\title{
Fast rotating massive stars and the origin of the abundance patterns in galactic globular clusters
}

\author{
T. Decressin ${ }^{1}$, G. Meynet ${ }^{1}$, C. Charbonnel ${ }^{1,2}$, N. Prantzos ${ }^{3}$, and S. Ekström ${ }^{1}$ \\ 1 Geneva Observatory, University of Geneva, chemin des Maillettes 51, 1290 Sauverny, Switzerland \\ e-mail: Thibaut.Decressin@obs.unige.ch \\ ${ }^{2}$ Laboratoire d'Astrophysique de Toulouse et Tarbes - UMR 5572 - Université Paul Sabatier Toulouse 3 - CNRS, 14 Av. E. Belin, \\ 31400 Toulouse, France \\ 3 Institut d'Astrophysique de Paris, CNRS UMR 7095, Univ. P. \& M. Curie, 98bis Bd. Arago, 75104 Paris, France
}

Received 11 July 2006 / Accepted 27 October 2006

\begin{abstract}
Aims. We propose the Wind of Fast Rotating Massive Stars scenario to explain the origin of the abundance anomalies observed in globular clusters.

Methods. We compute and present models of fast rotating stars with initial masses between 20 and $120 M_{\odot}$ for an initial metallicity $Z=$ $0.0005([\mathrm{Fe} / \mathrm{H}] \simeq-1.5)$. We discuss the nucleosynthesis in the H-burning core of these objects and present the chemical composition of their ejecta. We consider the impact of uncertainties in the relevant nuclear reaction rates.

Results. Fast rotating stars reach critical velocity at the beginning of their evolution and remain near the critical limit during the rest of the main sequence and part of the He-burning phase. As a consequence they lose large amounts of material through a mechanical wind which probably leads to the formation of a slow outflowing disk. The material in this slow wind is enriched in H-burning products and presents abundance patterns similar to the chemical anomalies observed in globular cluster stars. In particular, the $\mathrm{C}, \mathrm{N}, \mathrm{O}, \mathrm{Na}$ and $\mathrm{Li}$ variations are well reproduced by our model. However the rate of the ${ }^{24} \operatorname{Mg}(p, \gamma)$ has to be increased by a factor 1000 around $50 \times 10^{6} \mathrm{~K}$ in order to reproduce the amplitude of the observed $\mathrm{Mg}$-Al anticorrelation. We discuss how the long-lived low-mass stars currently observed in globular clusters could have formed out of the slow wind material ejected by massive stars.
\end{abstract}

Key words. nuclear reactions, nucleosynthesis, abundances - stars: rotation - stars: mass-loss - stars: abundances galaxies: clusters: general - galaxies: clusters: individual: NGC 6752

\section{Introduction}

Galactic globular clusters (hereafter GCs) appear to be chemically homogeneous (with the notable exception of $\omega$ Cen) with respect to the iron-group ( $\mathrm{Mn}, \mathrm{Fe}, \mathrm{Ni}, \mathrm{Cu})$, neutron-capture $(\mathrm{Ba}$, $\mathrm{La}, \mathrm{Eu})$ and alpha-elements (Si, Ca) (e.g., Kraft et al. 1992; James et al. 2004a,b; Sneden 2005; Sobeck et al. 2006). However it has long been known that these large aggregates of stars show strong inhomogeneities in lighter elements: $\mathrm{C}, \mathrm{N}, \mathrm{O}, \mathrm{Na}$, $\mathrm{Mg}$ and $\mathrm{Al}$ abundances show large star-to-star abundance variations within all the individual GCs studied up to now (for complete references see the early reviews by Freeman \& Norris 1981; Smith 1987; and Kraft 1994; and the more recent ones by Gratton et al. 2004; and Charbonnel 2005).

The observed patterns point to the simultaneous operation of the $\mathrm{CNO}, \mathrm{NeNa}$ and $\mathrm{MgAl}$ cycles of hydrogen burning: $\mathrm{C}$ and $\mathrm{N}, \mathrm{O}$ and $\mathrm{Na}$, and $\mathrm{Mg}$ and $\mathrm{Al}$ are respectively anticorrelated, the abundances of $\mathrm{C}, \mathrm{O}$ and $\mathrm{Mg}$ being depleted while those of $\mathrm{N}, \mathrm{Na}$ and $\mathrm{Al}$ are enhanced. Whenever $\mathrm{C}, \mathrm{N}$, and $\mathrm{O}$ are observed simultaneously, their sum appears to be constant within the observational errors (e.g., Dickens et al. 1991; Ivans et al. 1999). The sum $\mathrm{Mg}+\mathrm{Al}$ is also found to be constant in several clusters (Shetrone 1996). Observations in NGC 6752, M 13 and $\mathrm{M} 71$ show that the $\mathrm{Mg}$ depletion is due to the burning of ${ }^{24} \mathrm{Mg}$ while ${ }^{25} \mathrm{Mg}$ is untouched and ${ }^{26} \mathrm{Mg}$ is produced in the $\mathrm{Al}-$ rich stars (Yong et al. 2003, 2005, 2006). Li was found to be anticorrelated with Na in turn-off stars of NGC 6752 (Pasquini et al. 2005). All these features are considered anomalous because they are not seen in field stars of similar metallicity (e.g., Gratton et al. 2000).

For many years only the brightest GC red giants were accessible for detailed spectroscopic observations, and two main theoretical streams competed to explain the available data: (1) the so-called "evolution" scenario according to which the chemical anomalies are generated inside the low-mass stars we are presently observing, and (2) the "self-enrichment" (or primordial) scenario according to which such patterns pre-existed in the protocluster gas and were inherited at the birth of the longlived stars.

The evolution hypothesis has been seriously challenged by recent spectroscopic observations of less luminous stars in earlier stages of evolution in a number of GCs. Such studies revealed that stars located slightly above and below the main sequence turnoff exhibit the same anomalies as their giant counterparts (Gratton et al. 2001; Grundahl et al. 2002; Carretta et al. 2003, 2004; Cohen et al. 2002; Ramírez \& Cohen 2002, 2003; Harbeck et al. 2003). However such objects are not hot enough $^{1}$ for the required set of nuclear reactions to occur within their interior. While the $\mathrm{CNO}$ cycle is activated for temperatures

\footnotetext{
${ }^{1}$ In the central region of a $0.85 M_{\odot},[\mathrm{Fe} / \mathrm{H}]=-1.3$ turnoff star, the temperature is of the order of $25 \times 10^{6} \mathrm{~K}$.
} 
above $20 \times 10^{6} \mathrm{~K}$, the $\mathrm{NeNa}$ and $\mathrm{MgAl}$ chains require temperatures around $35 \times 10^{6} \mathrm{~K}$ and $50 \times 10^{6} \mathrm{~K}$ respectively. Destruction of ${ }^{24} \mathrm{Mg}$ by proton-capture needs still higher temperatures, around $70 \times 10^{6} \mathrm{~K}$ (e.g., Arnould et al. 1999; Prantzos $\&$ Charbonnel, in preparation). As a consequence, the abundance variations cannot be produced in situ, but certainly reflect the initial composition of the protostars. It is thus clear now that a large fraction of GC low-mass stars were formed from material processed through H-burning at high temperatures and then lost by more massive and faster evolving stars, and perhaps mixed with some original gas. Various aspects of this "self-enrichment scenario" are discussed in details by Prantzos \& Charbonnel (2006, hereafter PC06).

Regarding the nucleosynthetic site, most studies have focused on massive AGB stars which were suggested as the possible polluters by Cottrell \& Da Costa (1981). The two main reasons why these objects have been favored are that (1) they host regions where H-burning occurs at high temperatures (in particular when they experience the so-called hot bottom burning, or $\mathrm{HBB}$, at the base of their convective envelope between successive thermal pulses), and (2) the material they eventually eject (by stellar winds or Roche lobe overflow) is not enriched in iron. This last property is consistent with the observation above that the iron abundance in a GC does not show any significant scatter. For long, the AGB hypothesis was discussed only on a qualitative basis. However recent custommade stellar models (Ventura et al. 2001, 2002; Denissenkov \& Herwig 2003; Karakas \& Lattanzio 2003; Herwig 2004a,b; Ventura \& D'Antona 2005a-c, 2006; Decressin et al., in preparation) pointed out very severe difficulties from the nucleosynthesis point of view which stem from the competition between the HBB and the third dredge-up. This third dredge-up process does indeed contaminate the envelope of the AGB with the products of helium burning and creates abundance patterns in conflict with the observed ones (see Fenner et al. 2004; and Charbonnel 2005 for more details). PC06 discuss other shortcomings of the AGB scenario, the main one being related to the peculiar initial mass function it requires. In addition, they underline the fact that the AGB scenario gives no satisfactory answer as to the role of stars more massive and less massive than the presumed polluters.

Massive stars, more precisely, Wolf-Rayet stars, have been proposed by Brown \& Wallerstein (1993) and by Wallerstein et al. (1987) as possible sources for the very early enrichment of globular clusters. More recently Maeder \& Meynet (2006) suggested that He-rich stars in $\omega$ Cen could be formed from wind material of fast rotating massive stars. Prantzos \& Charbonnel (2006) proposed a comprehensive (albeit qualitative) scenario for the role of massive stars, suggesting that their winds provide the metal-enriched material for the next stellar generation, and that the subsequent supernova explosions provide the trigger for star formation; the SN ejecta escape the GC environment along the cavities opened previously by the stellar winds. PC06 also studied the massive star IMF required to explain quantitatively the number of Na-enhanced stars observed in NGC 2808 and found it to be flatter than canonical (i.e. Salpeter) IMFs; even flatter IMFs would be required if the polluters were AGB stars. Similar conclusions for a flat IMF are reached by Smith (2006), for the case of $\mathrm{N}$ enhancement of GC by massive star winds. The main reason why such objects have been discarded in the past in the context of the self-enrichment scenario is related to the fact that iron is ejected at the time their supernova explosion and this constitutes a priori a serious drawback for considering massive stars as GC pollution sources. This is true unless some filtering process removes the ejecta enriched by helium burning and more advanced nuclear stages while preserving those bearing the signatures of hydrogen processing. This is the key point of the Winds of Fast Rotating Massive Stars scenario (hereafter WFRMS) that we propose in the present work. In our framework, the GC chemical anomalies are built in H-burning zones of massive stars. Rotational mixing brings to the surface CNO-processed material which can then be ejected in a slow wind when the stars rotate at the critical limit.

As shown by Sackmann \& Anand (1970) and later by Langer (1998) and Maeder \& Meynet (2001), massive stars do reach the so-called critical velocity ${ }^{2}$ early on the main sequence if (1) they start their evolution with a sufficiently high initial rotation rate, (2) they do not lose too much angular momentum through stellar winds and (3) an efficient mechanism (meridional circulation in our models) transports angular momentum from the core to the envelope. Once the critical limit is reached, the surface velocity remains near the critical value during the rest of the main sequence and very likely an equatorial disk is formed as observed for instance around Be stars. In the case of these stars, only minor outflow in the line-forming region is observed (see the review by Porter \& Rivinius 2003). This material has thus a chance to be retained in the GC potential well. On the other hand fast rotation leads to strong internal mixing of the chemicals. As a result the ejected material will present signs of H-processing occurring in the stellar core. Thus if new stars form out of the slow wind material, their composition would bear the signatures of $\mathrm{H}$-burning. The main question addressed in this paper is whether the chemical composition of the ejecta of fast rotating massive stars is compatible with what observed in the long-lived GC stars.

In the present paper we develop in detail the WFRMS scenario. In Sect. 2 we describe the physical ingredients of our models of rotating massive stars. We first focus on the properties of the $60 M_{\odot}$ models computed with various assumptions. For this initial stellar mass we discuss the nucleosynthesis in the H-burning core in Sect. 3 while in Sect. 4 we investigate the chemical composition of the wind ejecta. The full range of masses between 20 and $120 M_{\odot}$ is investigated in Sect. 5. In Sect. 6 comparisons between the wind composition and the GC abundance patterns are performed. A schematic and speculative discussion of the complete scenario for explaining the inhomogeneities in GCs is presented in Sect. 7. The conclusions and some future lines of research are given in Sect. 8.

\section{Physical inputs}

Our stellar models were computed with the Geneva evolution code including the effects of rotation (Meynet \& Maeder 2000). We focus on the mass range corresponding to stars with high enough central temperatures on the main sequence for the $\mathrm{NeNa}$ and $\mathrm{MgAl}$ chains (see Fig. 1) to be activated. Models with initial masses of 20, 40, 60, and $120 M_{\odot}$ are computed from the Zero Age Main Sequence up to the end of the core He-burning phase, and a $200 M_{\odot}$ model is computed up to the end of the core H-burning phase. The theoretical predictions for the $60 M_{\odot}$ star are discussed in detail in Sects. 3 and 4 in order to properly illustrate the WFRMS scenario.

2 By critical velocity, we mean the equatorial surface velocity such that the centrifugal acceleration exactly balances the gravity. 


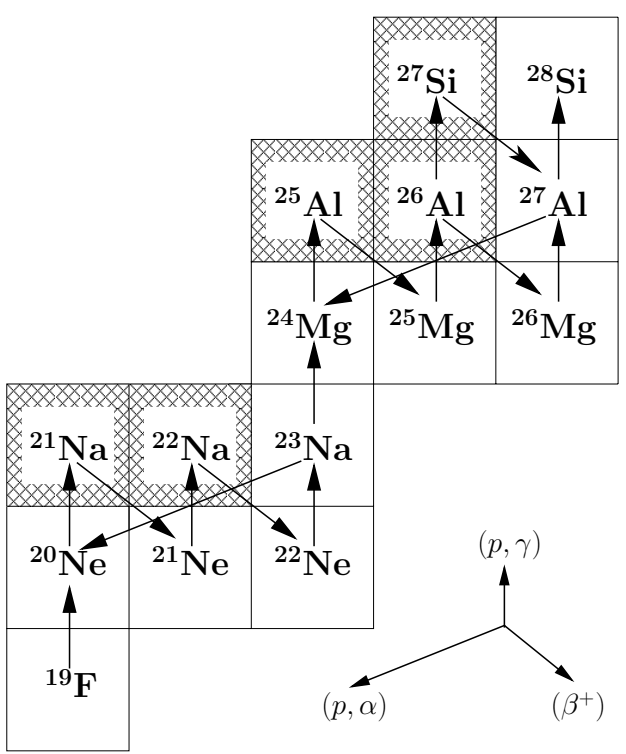

Fig. 1. Network of nuclear reactions involved in the $\mathrm{NeNa}$ and $\mathrm{MgAl}$ chains. Unstable nuclei are in shaded boxes. Arrows indicate the type of nuclear reactions: $(p, \gamma), \beta^{+}$, and $(p, \alpha)$.

\subsection{Microphysics}

We use the OPAL opacities (Iglesias \& Rogers 1996), complemented at temperatures below $5000 \mathrm{~K}$ with the molecular opacities of Alexander \& Ferguson (1994) (http: //webs . wichita.edu/physics/opacity).

In order to investigate the effects of the nuclear reaction rate uncertainties on the products of hydrogen nucleosynthesis, we present four models for the $60 M_{\odot}$ star computed using different sets of nuclear reactions for the hydrogen-burning network (see Table 1). Set A uses all the nominal values of the NACRE compilation (Angulo et al. 1999). The three other cases include the updates of Iliadis et al. (2001) and Hale et al. (2002, 2004) for the reactions involved in the $\mathrm{NeNa}$ and $\mathrm{MgAl}$ chains but with different options (see Table 2). Set B includes nominal values while in set $\mathrm{C}$ some specific rates are set to the experimental upper or lower limits. Figure 2 presents the corresponding rates for the temperature range between $30 \times 10^{6}$ and $80 \times 10^{6} \mathrm{~K}$ which is typical of the central temperatures of our main sequence stars. Set D is similar to set C except for the proton-capture on ${ }^{24} \mathrm{Mg}$ which is increased by three orders of magnitude compared to the Iliadis et al. (2001) nominal value at around $50 \times 10^{6} \mathrm{~K}$.

The initial composition of the chemical mixture is given in Table 3. It corresponds to that used to compute the opacity tables (Iglesias \& Rogers 1996, Weiss alpha-enhanced elements mixture). The metallicity of our models is $[\mathrm{Fe} / \mathrm{H}] \simeq-1.5$ corresponding to that of NGC 6752 which is the GC with the largest set of abundance data. The initial isotopic ratios of magnesium are taken equal to 80:10:10; this corresponds to the values observed in NGC 6752 "unpolluted" stars (i.e., in stars with high O and low Na abundances) in contrast with "polluted" stars which display large $\mathrm{O}$ depletion with high $\mathrm{Na}$ abundance (Yong et al. 2003, 2006).

\subsection{Rotation and mass loss}

We follow the formalism by Zahn (1992) and Maeder \& Zahn (1998) for the transport of angular momentum and chemicals in rotating stars. The effects of both meridional circulation and
Table 1. Main physical inputs of the stellar models for the various initial masses considered. The labels " $r$ " and "s" indicate respectively the models computed with or without rotation while the labels A-D refer to the choices in nuclear reactions (see text and Table 2). The initial value of $\Omega / \Omega_{\text {crit }}$ is given. [I], [H02] and [H04] correspond respectively to Iliadis et al. (2001) and Hale et al. (2002, 2004).

\begin{tabular}{cccl}
\hline \hline $\mathrm{M}\left(M_{\odot}\right)$ & Label & $\Omega / \Omega_{\text {crit }}$ & Nuclear rates \\
\hline 60 & $60 \mathrm{rA}$ & 0.95 & set $^{\dagger}:$ NACRE (nominal) \\
& $60 \mathrm{rB}$ & 0.95 & ${\text { set } \mathrm{B}^{\dagger}:[\mathrm{I}, \mathrm{H} 02, \mathrm{H} 04] \text { (nominal) }}$ \\
& $60 \mathrm{rC}$ & 0.95 & set $^{\dagger}:[\mathrm{I}, \mathrm{H} 02, \mathrm{H} 04]$ (exp. limits) \\
& $60 \mathrm{rD}$ & 0.95 & set $^{\dagger}$ \\
& $60 \mathrm{rE}$ & 0.80 & set C \\
\hline 20 & $20 \mathrm{rC}$ & 0.95 & set C \\
40 & $40 \mathrm{rC}$ & 0.98 & set C \\
120 & $120 \mathrm{rC}$ & 0.80 & set C \\
200 & $200 \mathrm{rC}$ & 0.95 & set C \\
20 & $20 \mathrm{sC}$ & 0 & set C \\
40 & $40 \mathrm{sC}$ & 0 & set C \\
60 & $40 \mathrm{sC}$ & 0 & set C \\
120 & $120 \mathrm{sC}$ & 0 & set C \\
\hline
\end{tabular}

${ }^{\dagger}$ Details on the nuclear rates used are presented in Table 2.

Table 2. Nuclear reaction rates adopted for the $\mathrm{NeNa}$ - and $\mathrm{MgAl}$-chains in the sets B-D. [N], [I], [H02] and [H04] correspond respectively to Angulo et al. (1999), Iliadis et al. (2001) and Hale et al. (2002, 2004). "Nom.", "low." and "up." refer respectively to nominal, lower and upper limits of the experimental values. For all the other reactions of our network we use the NACRE nominal values.

\begin{tabular}{ccccc}
\hline \hline Reaction & Set A & Set B & Set C & Set D \\
\hline${ }^{20} \mathrm{Ne}(p, \gamma)$ & {$[\mathrm{N}]$, nom. } & {$[\mathrm{N}]$, nom. } & {$[\mathrm{N}]$, low. } & {$[\mathrm{N}]$, low. } \\
${ }^{21} \mathrm{Ne}(p, \gamma)$ & {$[\mathrm{N}]$, nom. } & {$[\mathrm{I}]$, nom. } & {$[\mathrm{I}]$, low. } & {$[\mathrm{I}]$, low. } \\
${ }^{22} \mathrm{Ne}(p, \gamma)$ & {$[\mathrm{N}]$, nom. } & {$[\mathrm{H} 2]$, nom. } & {$[\mathrm{H} 2]$, low. } & {$[\mathrm{H} 2]$, low. } \\
${ }^{23} \mathrm{Na}(p, \gamma)$ & {$[\mathrm{N}]$, nom. } & {$[\mathrm{H} 4]$, nom. } & {$[\mathrm{H} 4]$, low. } & {$[\mathrm{H} 4]$, low. } \\
${ }^{23} \mathrm{Na}(p, \alpha)$ & {$[\mathrm{N}]$, nom. } & {$[\mathrm{H} 4]$, nom. } & {$[\mathrm{H} 4]$, up. } & {$[\mathrm{H} 4]$, up. } \\
${ }^{24} \mathrm{Mg}(p, \gamma)$ & {$[\mathrm{N}]$, nom. } & {$[\mathrm{I}]$, nom. } & {$[\mathrm{I}]$, up. } & {$[\mathrm{I}],+3$ dex } \\
${ }^{25} \mathrm{Mg}(p, \gamma)$ & {$[\mathrm{N}]$, nom. } & {$[\mathrm{I}]$, nom. } & {$[\mathrm{I}]$, up. } & {$[\mathrm{I}]$, up. } \\
${ }^{26} \mathrm{Mg}(p, \gamma)$ & {$[\mathrm{N}]$, nom. } & {$[\mathrm{I}]$, nom. } & {$[\mathrm{I}]$, up. } & {$[\mathrm{I}]$, up. } \\
${ }^{27} \mathrm{Al}(p, \gamma)$ & {$[\mathrm{N}]$, nom. } & {$[\mathrm{I}]$, nom. } & {$[\mathrm{I}]$, low. } & {$[\mathrm{I}]$, low. } \\
${ }^{27} \mathrm{Al}(p, \alpha)$ & {$[\mathrm{N}]$, nom. } & {$[\mathrm{I}]$, nom. } & {$[\mathrm{I}]$, low. } & {$[\mathrm{I}]$, low. } \\
\hline
\end{tabular}

shear turbulence are taken into account: the meridional circulation advects angular momentum and the shear acts as a diffusive process. The transport of chemical species is computed as a diffusive process as the result of meridional circulation and horizontal and vertical turbulence (Chaboyer \& Zahn 1992). The treatment of the convective instability is done according to the Schwarzschild criterion and we do not consider overshooting.

The treatment of rotation includes the hydrostatic effects following Meynet \& Maeder (1997) as well as the impact of rotation on the mass loss rate described by Maeder \& Meynet (2000). We do not account for the wind anisotropies induced by rotation as in Maeder (1999) although the related effects would reinforce the trends found in this paper by fastening the arrival at the break-up limit (see Sect. 4.1).

The radiative mass loss rates are from Kudritzki \& Puls (2000) when $\log T_{\text {eff }}>3.95$ and from de Jager et al. (1988) otherwise. When a model reaches the WR phase (i.e., when the surface hydrogen mass fraction becomes lower than 0.4 and the effective temperature is higher than $10^{4} \mathrm{~K}$ ), the mass loss rate is switched to the prescription of Nugis \& Lamers (2000). Except for the WR phase, we consider a dependence of the mass loss 

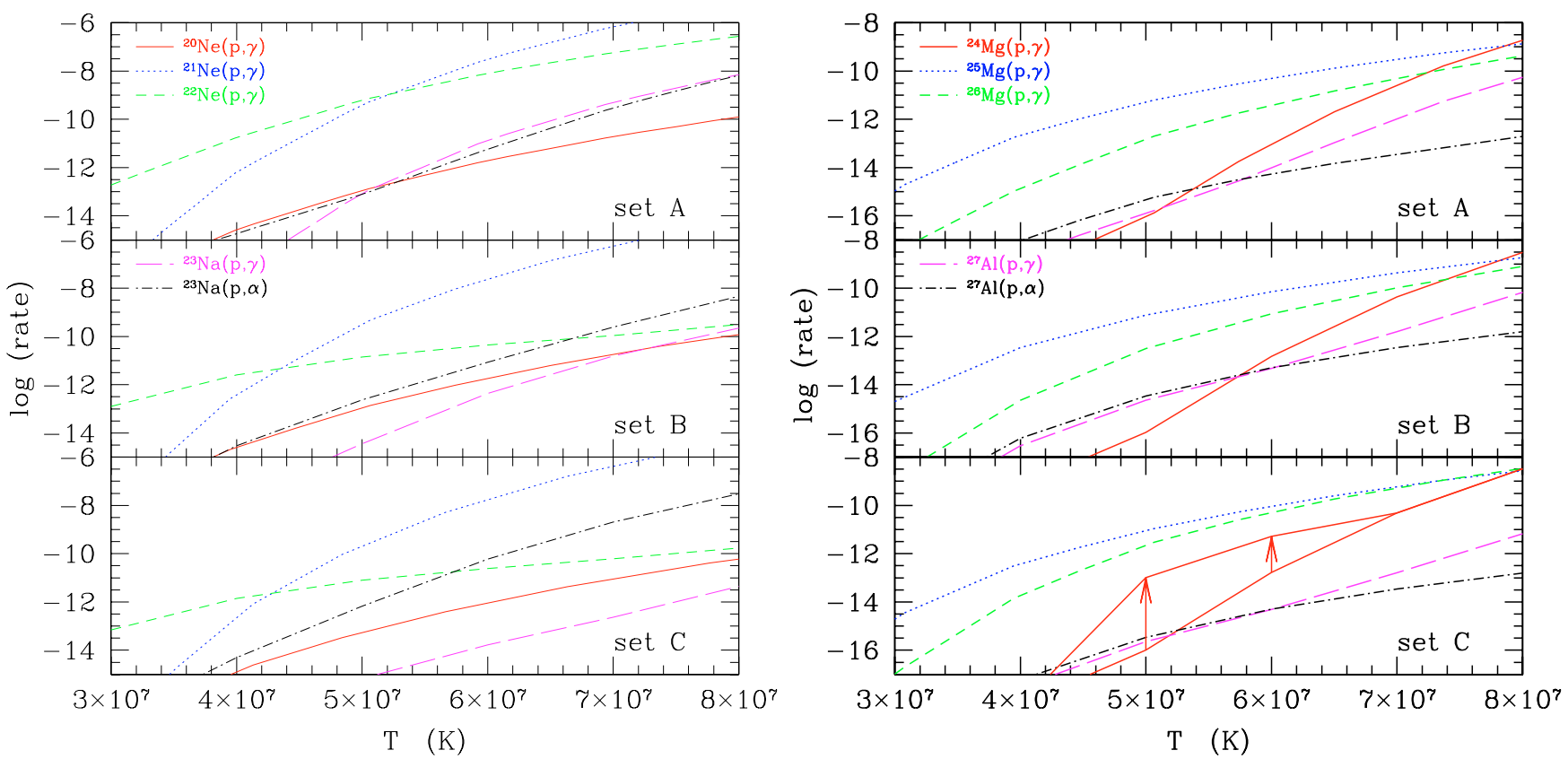

Fig. 2. Nuclear reaction rates for $\mathrm{NeNa}(l e f t)$ and $\mathrm{MgAl}$ chains (right) from the set $\mathrm{A}, \mathrm{B}$ and $\mathrm{C}$ (top to bottom). Arrows and upper full line in the lower right panel indicate the increase of the rate of ${ }^{24} \mathrm{Mg}(\mathrm{p}, \gamma)^{25} \mathrm{Al}$ at $T=50$ and $60 \times 10^{6} \mathrm{~K}$ assumed in set $\mathrm{D}$.

Table 3. Initial abundances in mass fraction.

\begin{tabular}{cccc}
\hline \hline Element & Abundance & Element & Abundance \\
\hline${ }^{1} \mathrm{H}$ & 0.754 & ${ }^{19} \mathrm{~F}$ & $1.53 \mathrm{e}-8$ \\
${ }^{3} \mathrm{He}$ & $2.93 \mathrm{e}-5$ & ${ }^{20} \mathrm{Ne}$ & $5.30 \mathrm{e}-5$ \\
${ }^{4} \mathrm{He}$ & 0.245 & ${ }^{21} \mathrm{Ne}$ & $5.00 \mathrm{e}-8$ \\
${ }^{12} \mathrm{C}$ & $3.50 \mathrm{e}-5$ & ${ }^{22} \mathrm{Ne}$ & $4.72 \mathrm{e}-6$ \\
${ }^{13} \mathrm{C}$ & $1.47 \mathrm{e}-7$ & ${ }^{23} \mathrm{Na}$ & $3.30 \mathrm{e}-7$ \\
${ }^{14} \mathrm{~N}$ & $1.03 \mathrm{e}-5$ & ${ }^{24} \mathrm{Mg}$ & $1.68 \mathrm{e}-5$ \\
${ }^{15} \mathrm{~N}$ & $1.58 \mathrm{e}-8$ & ${ }^{25} \mathrm{Mg}$ & $2.10 \mathrm{e}-6$ \\
${ }^{16} \mathrm{O}$ & $3.00 \mathrm{e}-4$ & ${ }^{26} \mathrm{Mg}$ & $2.10 \mathrm{e}-6$ \\
${ }^{17} \mathrm{O}$ & $1.31 \mathrm{e}-7$ & ${ }^{27} \mathrm{Al}$ & $9.00 \mathrm{e}-7$ \\
${ }^{18} \mathrm{O}$ & $7.45 \mathrm{e}-6$ & ${ }^{28} \mathrm{Si}$ & $2.56 \mathrm{e}-5$ \\
\hline
\end{tabular}

rates with metallicity as $\dot{M} \propto \sqrt{Z / Z_{\odot}}$, where $Z$ is the mass fraction of heavy elements at the surface of the star.

As in Meynet et al. (2006) a specific treatment for mass loss has been applied at break-up. According to Meynet \& Maeder (2000) three kinds of "break-up limits" can be defined depending on which mechanism (i.e., radiative acceleration, centrifugal acceleration or both) contributes to counterbalance the gravity: 1 The $\Gamma$-Limit, when radiation effects largely dominate; 2 The $\Omega$-Limit, when rotation effects determine break-up; 3 The $\Omega \Gamma$-Limit, when both rotation and radiation are important for the critical velocity. In the present work the $\Omega$-Limit is reached during the MS, while the $\Omega \Gamma$-Limit is encountered by our most massive stellar models $\left(M \geq 60 M_{\odot}\right)$ just after the MS.

During the MS, near the $\Omega$-Limit, two counteracting effects compete. On one hand, matter is removed from the stellar surface (mainly through the equatorial regions) together with angular momentum. Also the expansion of the envelope tends to slow down the surface. On the other hand, meridional advection in the outer layers acts so as to transfer angular momentum from the inner stellar regions to the surface (see for instance Fig. 1 in Meynet \& Maeder 2002). This acts as to accelerate the surface. As long as the internal transport of angular momentum is efficient enough for accelerating the outer layers in a timescale shorter than the mass loss or inflation timescale, the surface velocity remains near the critical limit. In practice, however, the critical limit contains mathematical singularities; we thus consider that during the break-up phase, the mass loss rate is such that the rotation velocity stays near a constant fraction of the critical value (0.98 typically). At the end of the main sequence, the stellar radius inflates so rapidly that meridional circulation cannot anymore ensure the internal coupling and the break-up phase ceases naturally. However the most massive stellar models encounter the $\Omega \Gamma$-Limit after the MS. In that case, we apply similar procedures as the one described above, i.e. we apply mass loss rates that maintain the model at a constant distance from the $\Omega \Gamma$-Limit. When the star has lost a sufficient amount of mass to evolve away from this limit, the regular mass loss rates apply again.

We explore the case of high initial rotational velocities. For the stars with initial mass between 40 and $120 M_{\odot}$ we take $V_{\text {ini }}=$ $800 \mathrm{~km} \mathrm{~s}^{-1}$. The $200 M_{\odot}$ model starts with $V_{\text {ini }}=1000 \mathrm{~km} \mathrm{~s}^{-1}$. This corresponds to an initial value of $\Omega / \Omega_{\text {crit }}$ between 0.80 and 0.98 . The $20 M_{\odot}$ star starts with $V_{\text {ini }}=600 \mathrm{~km} \mathrm{~s}^{-1}$ as it nearly corresponds to the break-up velocity $\left(\Omega / \Omega_{\text {crit }}=0.95\right)$.

\section{Central hydrogen-burning in a $60 M_{\odot}$ star}

As a prerequisite for our scenario we check whether the abundance patterns due to nuclear reactions in the hydrogen-burning core of massive stars mimic the chemical trends observed in GC low-mass stars. Obviously the abundance variations obtained within the central regions are the most extreme that one can expect within the WFRMS scenario. Indeed some dilution of this processed material is expected in the radiative envelope of the polluters (see Sect. 4) and then eventually later with the intracluster gas (see Sect. 6). We are thus looking for stronger abundance variations in the stellar core than the presently observed for the elements involved in the $\mathrm{CNO}, \mathrm{NeNa}$ and $\mathrm{MgAl}$ cycles.

We first investigate the nucleosynthesis that occurs within the convective core of a main sequence $60 M_{\odot}$ star and we explore in detail the uncertainties of the nuclear reaction rates. The 


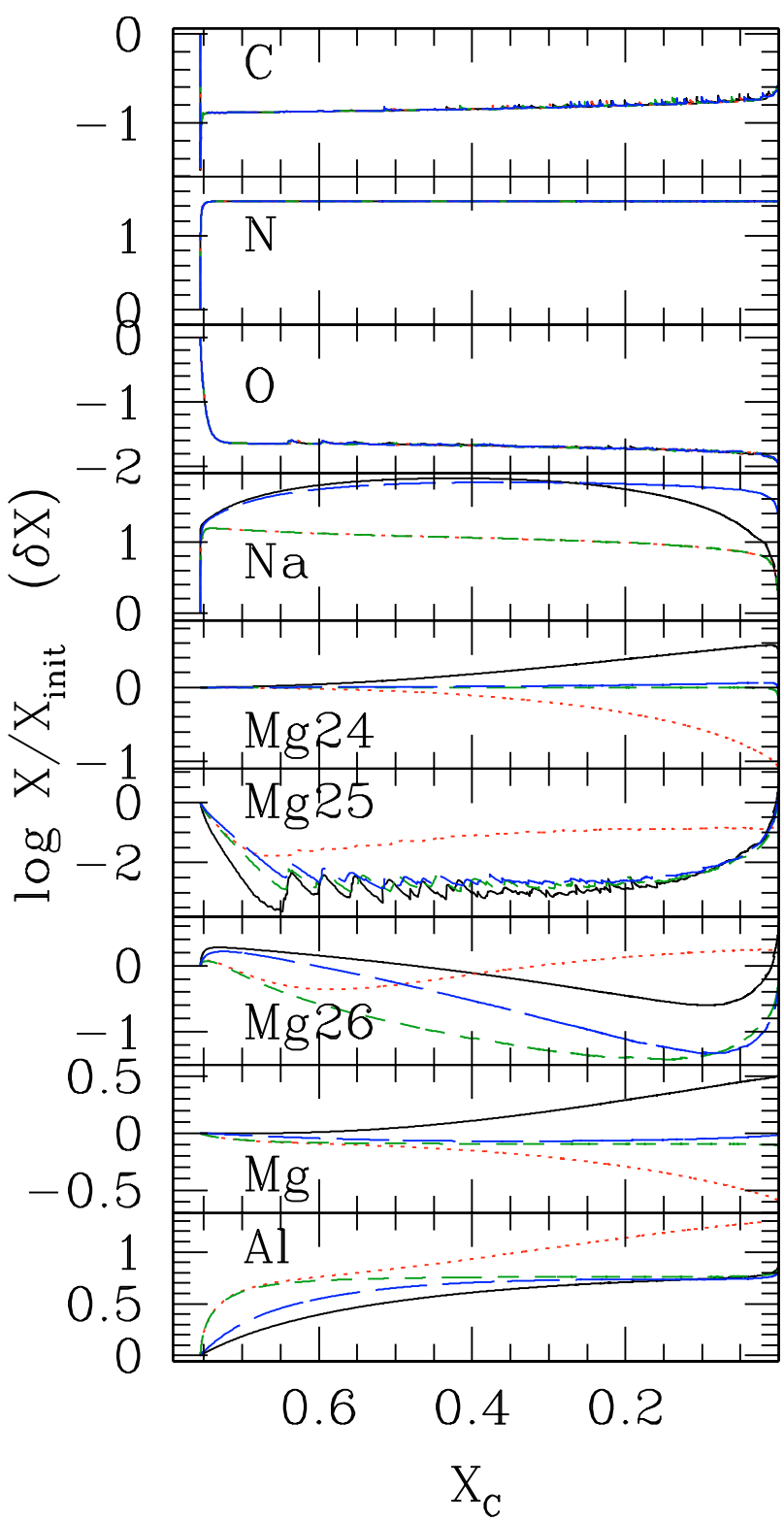

Fig. 3. Evolution of the central abundances in the $60 M_{\odot}$ rotating models computed with different sets of nuclear reaction rates: set A (full line), set B (long dashed lines), set C (short dashed lines) and set D (dotted lines). The amount of the unstable nucleus ${ }^{26} \mathrm{Al}$ is added to those of ${ }^{26} \mathrm{Mg}$ and $\mathrm{Mg}$.

evolution of the central abundances of the key elements is presented in Fig. 3 as a function of the central abundance of hydrogen for the various sets of nuclear reactions (see Table 2).

\subsection{CNO nucleosynthesis}

In the $60 M_{\odot}$ star the central temperature on the main sequence varies from $48 \times 10^{6} \mathrm{~K}$ to $75 \times 10^{6} \mathrm{~K}$ (see Fig. 7). The $\mathrm{CNO}$ cycle thus rapidly reaches equilibrium at the beginning of $\mathrm{H}$-burning. As a result the central abundance of ${ }^{12} \mathrm{C}$ drops suddenly by about an order of magnitude, while that of ${ }^{14} \mathrm{~N}$ increases by a factor of 29 . Slightly later ${ }^{16} \mathrm{O}$ reaches its equilibrium value, which is 1.6 dex below its initial abundance. After this adjustment phase at the beginning of the main sequence, the CNO elements stay at their equilibrium level. These predictions are identical for all the $60 M_{\odot}$ models presented here because they were all computed with NACRE prescriptions for the CNO-cycle reaction rates (no modification of these rates has been published since the NACRE compilation was made available).

\subsection{NeNa and MgAl predictions with the NACRE nominal values - Model 60rA}

Model 60rA was computed with the NACRE nominal values for the whole nuclear network (full lines in Fig. 3).

As can be seen in Fig. 3, the central abundance of ${ }^{23} \mathrm{Na}$ shows a three step evolution on the main sequence: a rapid raise by 1.2 dex, then a more progressive increase of 0.7 dex until the mass fraction of hydrogen at the center, $X_{\mathrm{c}}$, equals 0.4 , and finally a decrease. The initial feature is due to proton-captures on ${ }^{21} \mathrm{Ne}$ and ${ }^{22} \mathrm{Ne}$ which are the fastest reactions of the NeNa chain (see Fig. 2). As proton-capture on ${ }^{23} \mathrm{Na}$ is slower, the abundance of sodium increases until the complete consumption of ${ }^{21} \mathrm{Ne}$ and ${ }^{22} \mathrm{Ne}$. Later on ${ }^{20} \mathrm{Ne}$ starts burning in favor of sodium. The slow increase is due to the competition between the burning of ${ }^{20} \mathrm{Ne}$ and that of ${ }^{23} \mathrm{Na}$ via the channels $(p, \gamma)$ or $(p, \alpha)$. When using NACRE nominal values the transformation of ${ }^{20} \mathrm{Ne}$ into ${ }^{23} \mathrm{Na}$ is more efficient than the ${ }^{23} \mathrm{Na}$ destruction for $T$ inferior to $\sim 50 \times 10^{6} \mathrm{~K}$ which results in a slow increase of ${ }^{23} \mathrm{Na}$. When the temperature exceeds $50 \times 10^{6} \mathrm{~K}$ the situation reverses and the abundance of ${ }^{23} \mathrm{Na}$ decreases.

Let us now turn our attention to $\mathrm{Mg}$ and $\mathrm{Al}$. In model 60rA the central abundance of ${ }^{24} \mathrm{Mg}$ increases slightly during the main sequence. That of ${ }^{25} \mathrm{Mg}$ first decreases by nearly 4 dex, then it presents a sawtooth behavior until $X_{\mathrm{c}}$ equals $\sim 0.2$ and finally it increases. ${ }^{26} \mathrm{Mg}$ first slightly increases before decreasing during most of the core H-burning phase and increasing again when $X_{\mathrm{c}}$ becomes lower than $\sim 0.07$. The behavior of $\mathrm{Mg}$ (total) follows mainly that of ${ }^{24} \mathrm{Mg}$. Also the abundance of $\mathrm{Al}$ increases.

The increase of the ${ }^{24} \mathrm{Mg}$ abundance is due to protoncaptures on ${ }^{23} \mathrm{Na}$. The very efficient destruction of ${ }^{25} \mathrm{Mg}$ is due to the reaction ${ }^{25} \mathrm{Mg}(p, \gamma){ }^{26} \mathrm{Al}$. The sawtooth behavior results from small instabilities affecting the size of the convective core ${ }^{3}$. The slight production of this element at the end of the main sequence is due to the proton-captures on ${ }^{24} \mathrm{Mg}$. The initial raise of the abundance of ${ }^{26} \mathrm{Mg}$ results from the destruction of ${ }^{25} \mathrm{Mg}$ described above ${ }^{4}$. When all the ${ }^{25} \mathrm{Mg}$ is consumed within the core, ${ }^{26} \mathrm{Mg}$ is converted into aluminum.

Table 4 gives the mean variations of the central abundances during the main sequence for the NACRE nominal reaction rates. $\mathrm{O}$ and $\mathrm{Na}$ are respectively depleted and produced as required by the data. On the other hand the increase of the total magnesium we obtain is at odds with the observed $\mathrm{Mg}$ - $\mathrm{Al}$ anticorrelation. These results agree with those of Arnould et al. (1999) who investigated in detail hydrogen-nucleosynthesis at constant temperature and solar metallicity with NACRE reaction rates. They found a production of $\mathrm{Na}$ and they argue that it is dominated by uncertainties on the rates for $T>50 \times 10^{6} \mathrm{~K}$. Also ${ }^{24} \mathrm{Mg}$ is not destroyed even when experimental errors on rates are taken into account for $T<70 \times 10^{6} \mathrm{~K}$. Production of aluminum is due to the burning of both ${ }^{25} \mathrm{Mg}$ and ${ }^{26} \mathrm{Mg}$.

${ }^{3}$ When the size of the convective core slightly increases, some ${ }^{25} \mathrm{Mg}$ is dredged down. For most elements, the quantity dredged down is small compared to the abundance in the core; however in the case of ${ }^{25} \mathrm{Mg}$ which is severely depleted in the core, this temporarily produces a small increase in the central abundance.

${ }^{4}$ The abundance of the unstable isotope ${ }^{26} \mathrm{Al}$ which decays into ${ }^{26} \mathrm{Mg}$ is included in the total ${ }^{26} \mathrm{Mg}$ abundance. 


\section{3. $\mathrm{NeNa}$ and $\mathrm{MgAl}$ predictions with the Illiadis nominal values - Model 60rB}

In model 60rB we take into account the nominal rates given by Iliadis et al. (2001) and Hale et al. (2002, 2004) for the reactions involved in the $\mathrm{NeNa}$ and $\mathrm{MgAl}$ chains. The differences with the NACRE prescriptions can be seen in Fig. 2. At $T=50 \times 10^{6} \mathrm{~K}$ the rates of the ${ }^{22} \mathrm{Ne}(p, \gamma)$ and ${ }^{23} \mathrm{Na}(p, \gamma)$ reactions are lowered respectively by 1.7 dex and 1.3 dex compared to NACRE nominal values, while the rate of ${ }^{23} \mathrm{Na}(p, \alpha)$ is 0.6 dex higher. For ${ }^{27} \mathrm{Al}(p, \gamma)$ the new rate is increased by 1 dex at $T=50 \times 10^{6} \mathrm{~K}$, but is unchanged for $T>70 \times 10^{6} \mathrm{~K}$. Finally the rate of the ${ }^{27} \mathrm{Al}(p, \alpha){ }^{24} \mathrm{Mg}$ reaction is now higher by 0.7 to $1.1 \mathrm{dex}$ in the range of temperature of interest. The prescriptions for the other reactions are unchanged.

This update does not affect the global structure and evolution of the star, as the energizing rates concern the $\mathrm{CNO}$ cycle which is not modified. Thus the lifetime, the size of the convective core as well as the mass loss history are identical in all our models.

The key reaction for the $\mathrm{NeNa}$ chain is ${ }^{20} \mathrm{Ne}(p, \gamma)$ as ${ }^{20} \mathrm{Ne}$ is the most abundant neon isotope and its destruction by protoncaptures is much slower than that of the ${ }^{21} \mathrm{Ne}$ and ${ }^{22} \mathrm{Ne}$ (by 2.7 and 3.7 orders of magnitude respectively). Since the rate of this reaction is unchanged the behavior of the $\mathrm{Ne}$ isotopes is barely affected with respect to the results presented in Sect. 3.2. On the other hand because the rate of proton-capture on ${ }^{23} \mathrm{Na}$ is reduced now with respect to the former case, the consumption of sodium is lowered (its abundance does not decrease at the end of the main sequence). As a consequence the abundances of ${ }^{24} \mathrm{Mg}$ and of the total magnesium do not increase although one does not obtain the decrease required by the observations. Finally the ${ }^{26} \mathrm{Mg}$ burning is favored so that aluminum is produced earlier on the main sequence although its final abundance reaches the same value as in model 60rA.

In summary the new set of nuclear reactions partly resolves the difficulties encountered by model 60rA regarding the anticorrelation between $\mathrm{Mg}$ and $\mathrm{Al}$. However the predicted magnesium isotopic ratios are still in conflict with the data by Yong et al. (2003, 2005).

\subsection{NeNa and MgAl predictions with experimental extreme values - Model 60rC}

To solve the remaining difficulties, we look now for the most favorable set of nuclear reactions by considering the published experimental limits. In model 60rC (set C) we make the following assumptions (see Table 2): We take the lower limits for the burning rates of all the neon isotopes in order to lower the overall production of sodium. We also take the lower limit for the ${ }^{23} \mathrm{Na}(p, \gamma)$ reaction which now becomes the slowest reaction of the NeNa chain. This reduces the linkage between the two chains and disfavors the production of ${ }^{24} \mathrm{Mg}$. Regarding the $\mathrm{MgAl}$ chains, we take the upper limits for the destruction rates of the magnesium isotopes and the lower limits for the burning rates of ${ }^{27} \mathrm{Al}$.

The corresponding predictions are shown by the shortdashed lines in Fig. 3. The overall increase of the ${ }^{23} \mathrm{Na}$ abundance is more modest than in the previous models. One obtains now an important decrease of the ${ }^{26} \mathrm{Mg}$ abundance, while the final predictions for ${ }^{25} \mathrm{Mg}$ and ${ }^{27} \mathrm{Al}$ are essentially not affected relative to sets A-B. Again, ${ }^{24} \mathrm{Mg}$ stays constant during the hydrogen-burning phase. Nevertheless the total magnesium abundance decreases by 0.1 dex in this case. The stronger variation observed could come from more massive stars where the
Table 4. Mean core abundance variations during MS of the $60 M_{\odot}$ models. $\delta \mathrm{X}$ refers to the amplitude (in dex) of variation of element $X$. For NGC 6752 it refers to the amplitude variation observed between polluted and unpolluted stars.

\begin{tabular}{ccccccc}
\hline \hline Model & $\delta \mathrm{C}$ & $\delta \mathrm{N}$ & $\delta \mathrm{O}$ & $\delta \mathrm{Na}$ & $\delta \mathrm{Mg}$ & $\delta \mathrm{Al}$ \\
\hline NGC 6752 & -0.7 & 1.7 & -1.0 & 0.9 & -0.3 & 1.4 \\
\hline 60rA & -0.9 & 1.5 & -1.8 & 1.6 & 0.5 & 0.7 \\
60rB & -0.9 & 1.5 & -1.8 & 1.6 & 0.0 & 0.7 \\
60rC & -0.9 & 1.5 & -1.8 & 1.0 & -0.1 & 0.7 \\
60rD & -0.9 & 1.5 & -1.8 & 1.0 & -0.4 & 1.3 \\
\hline
\end{tabular}

central temperature is hotter and the burning rate of ${ }^{24} \mathrm{Mg}$ is faster (see Sect. 5).

\subsection{Model 60rD}

As shown previously the difficulty regarding the $\mathrm{Mg}$ destruction comes mainly from the fact that the central temperature reaches the required extreme values only at the very end of the main sequence (see Fig. 7). We thus note that the use of the rate published for the ${ }^{24} \mathrm{Mg}(p, \gamma)$ reaction (Powell et al. 1999) is not compatible with significant $\mathrm{Mg}$ depletion within the core of massive main sequence stars. This led us to tentatively modify this rate in order to reconcile the theoretical predictions with the abundance data.

In model 60rD we keep all the reaction rates as in $60 \mathrm{rC}$ except for the burning rate of ${ }^{24} \mathrm{Mg}$ that we artificially enhance by a factor of $10^{3}$ and $10^{1.5}$ at 50 and $60 \times 10^{6} \mathrm{~K}$ respectively (the recommended rate is used for temperature lower than $40 \times$ $10^{6} \mathrm{~K}$ and higher than $70 \times 10^{6} \mathrm{~K}$ ). With these assumptions this reaction becomes as efficient as the other ones involved in the $\mathrm{MgAl}$ chain (see the arrows in Fig. 2).

As a result (see the dotted lines in Fig. 2) the ${ }^{24} \mathrm{Mg}$ abundance decreases by about 0.4 dex when the central hydrogen mass fraction equals $\sim 0.2$. At the end of the main sequence ${ }^{25} \mathrm{Mg}$ and ${ }^{26} \mathrm{Mg}$ are respectively slightly destroyed and produced. Simultaneously the total magnesium abundance decreases by 0.3 dex while that of ${ }^{27} \mathrm{Al}$ increases by 1.2 dex for a central hydrogen mass of 0.2 . These predictions are in good agreement with the observational constraints.

\subsection{Summary}

Our models were computed with the metallicity of NGC 6752 $([\mathrm{Fe} / \mathrm{H}]=-1.5)$ to compare our theoretical predictions with the observations in this GC. In this section we have focused on the nucleosynthesis within the core of a $60 M_{\odot}$ main sequence star. The corresponding abundance variations are thus the most extreme one might expect, and they cannot be compared directly with the observational data. However it is a possible candidate the observed inhomogeneities and that it is worthwhile to explore further the WFRMS scenario.

In Table 4 we list the most extreme abundance variations between polluted and unpolluted stars in NGC 6752 as well as in the core of our $60 M_{\odot}$ models. The comparison supports the $\mathrm{O}-\mathrm{Na}$ anticorrelation and sets $\mathrm{A}$ and $\mathrm{B}$ lead to higher Na production than set $\mathrm{C}$. On the other hand, the $\mathrm{Mg}$-Al anticorrelation appears to be more difficult to reproduce. The use of sets A and $\mathrm{B}$ lead to a $\mathrm{Mg}$ - $\mathrm{Al}$ correlation, while that of set $\mathrm{C}$ builds up a weak $\mathrm{Mg}-\mathrm{Al}$ anticorrelation. The situation becomes more favorable when one increases the rate of the ${ }^{24} \mathrm{Mg}(p, \gamma){ }^{25} \mathrm{Al}$ reaction as in model $60 \mathrm{rD}$. In that case ${ }^{24} \mathrm{Mg}$ is destroyed while the 
abundance of ${ }^{25} \mathrm{Mg}$ is barely affected and that of ${ }^{26} \mathrm{Mg}$ slightly increases. This is in agreement with the observational constraints obtained for the $\mathrm{Mg}$ isotopes in NGC 6752 stars by Yong et al. (2005).

The H-burning signatures we describe are similar to those found in the H-burning shell during central He-burning. In this shell the temperature does not exceed $60 \times 10^{6} \mathrm{~K}$. Also the situation is not very different in rotating and non rotating models, the central temperatures and densities being weakly affected by rotation.

This study emphasizes the importance of some reactions: proton-captures on ${ }^{20} \mathrm{Ne}$ and ${ }^{23} \mathrm{Na}$ respectively govern the amount of sodium produced and the linkage between the $\mathrm{NeNa}$ and $\mathrm{MgAl}$ chains. Concerning the $\mathrm{MgAl}$ chain, the key reaction is the proton-capture on ${ }^{24} \mathrm{Mg}$. A raise of its rate with respect to the published values is required to reproduce the extreme variations of magnesium and aluminum in the central region of a $60 M_{\odot}$ star. This is due to the fact that the required temperature (of about $72-78 \times 10^{6} \mathrm{~K}$, see Prantzos \& Charbonnel in preparation) is reached in the stellar core only at the very end of the main sequence in this object (see Fig. 7).

\section{Mixing and ejection of matter in a rotating $60 M_{\odot}$ star}

In the previous section we discussed what happens from the nucleosynthetic point of view in the core of a (rotating or nonrotating) $60 M_{\odot}$ star. We will now describe the critical effects of rotation on stellar mass loss and internal chemical structure. Finally we will follow the evolution of the surface abundances and compare our predictions with the data in GC stars.

\subsection{Mass loss}

Figure 4 shows the evolution with time of the ratio between the surface and the break-up velocity in our $60 M_{\odot}$ model with $V_{\text {ini }}=800 \mathrm{~km} \mathrm{~s}^{-1}$. This ratio lies around 0.95 at the beginning of the main sequence. It rapidly drops to 0.91 due to the establishment of differential rotation in the star: during this initial phase angular momentum is carried from the external regions into the internal layers and the core accelerates (see Meynet \& Maeder 2000 ). Later on $\Omega / \Omega_{\text {crit }}$ increases up to reach unity (see Sect. 2 ). However we force the model to stay below this level in order to avoid numerical difficulties (see Sect. 2.2). Figure 4 shows that all the episodes of strong mass loss correspond to a decrease of the surface velocity below the critical value. Then surface rotation velocity raises again. This process probably leads to the formation of a circumstellar disk. We make the hypothesis that this material is eventually lost by the star.

During the whole main sequence, mass loss is dominated by this strong rotation-induced mechanical wind and is increased by more than a factor of 24 with respect to the standard case (e.g., model $60 \mathrm{rC}$ loses $20 M_{\odot}$ whereas the non rotating model expels less than $1 M_{\odot}$ ).

As we just described, fast rotation does change the stellar mass loss quantitatively. But it also has a crucial qualitative impact on the properties and the topology of the ejecta. When the star rotates close to or at break-up the centrifugal force balances gravity. As a result, the mass loss is at least partly mechanicallydriven, and the equatorial matter is released into a Keplerian disk. Very likely an equatorial disk forms as observed around Be stars (e.g., Porter \& Rivinius 2003). This ejected material will thus be very easily retained within the GC potential well. This

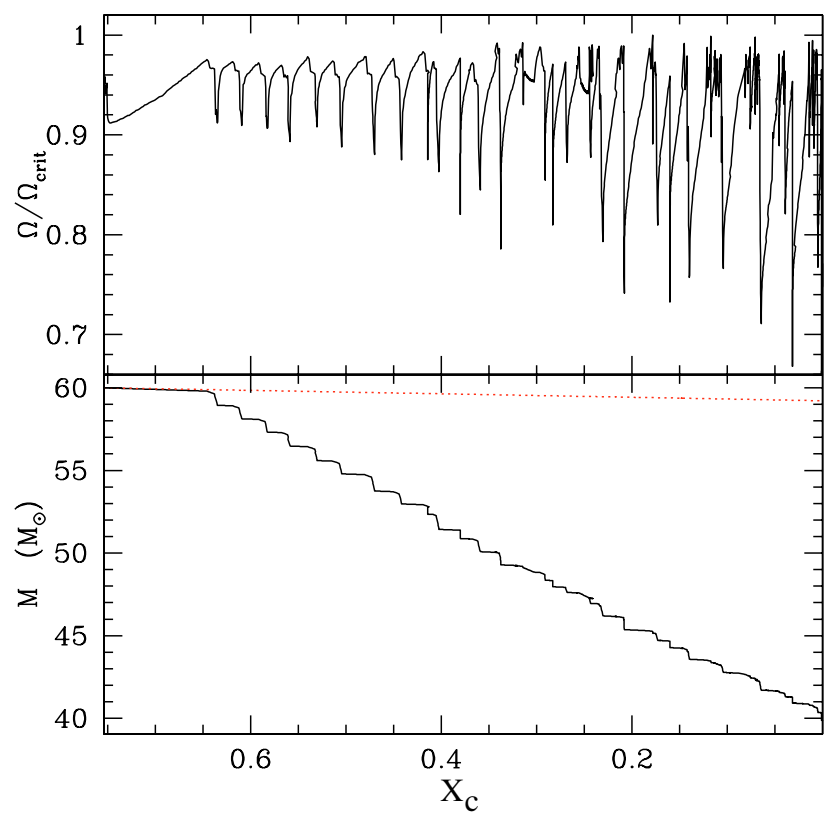

Fig. 4. (Top) Ratio of the surface rotation velocity to the break-up value for model 60rC. (Bottom) Evolution of the total stellar mass for models $60 \mathrm{rC}$ and $60 \mathrm{sC}$ (full and dotted lines respectively) along the main sequence (the abscissa is the central mass fraction of hydrogen).

is very different from the non-rotating situation where the radiative winds escape at high velocity. This is one of the key points of our scenario which will be discussed in detail in Sect. 7.

As explained in Sect. 2.2 the model evolves away from the $\Omega$-Limit when it leaves the MS. At that moment however the most massive stars $\left(M \geq 60 M_{\odot}\right)$ encounter the $\Omega \Gamma$-Limit. In the present work, we suppose that the matter lost at the $\Omega \Gamma$-Limit is released in an equatorial disk, as in the case of the material lost at the $\Omega$-Limit. When the star moves away from the $\Omega \Gamma$-Limit due to heavy mass loss, the radiatively-driven fast winds take over.

\subsection{Abundance profiles}

Since mass loss is very efficient in the rotating case, the outer layers of the star peel off revealing the $\mathrm{H}$-processed layers at the surface. Rotational mixing strengthens the modifications of the surface abundances as the products of central burning are transported outwards by various instabilities induced by rotation ${ }^{5}$.

In Fig. 5 we show the abundance profiles of various chemical elements as a function of the Lagrangian mass for the $60 M_{\odot}$ star (computed using set $\mathrm{C}$ for the nuclear network) at the end of the main sequence in the standard and rotating models $\left(V_{\text {ini }}=0\right.$ and $800 \mathrm{~km} \mathrm{~s}^{-1}$ respectively). In both cases the size of the convective core is very similar (respectively 23.7 and $23.5 M_{\odot}$ in the standard and rotating models at the end of the main sequence) as are the final central abundances (the central temperature differs by less than $1 \%$ between the two models).

Outside the convective core however the abundance profiles show some notable differences. The most striking effect is due

5 The most important ones are the meridional currents and the shear turbulence (see the review by Talon 2004). The inclusion of these transport mechanisms has improved the massive star models in many respects (see Maeder \& Meynet 2000, for references); in particular, rotating models can reproduce the chemical enrichment observed at the surface of OBA stars (Heger \& Langer 2000; Meynet \& Maeder 2000). 

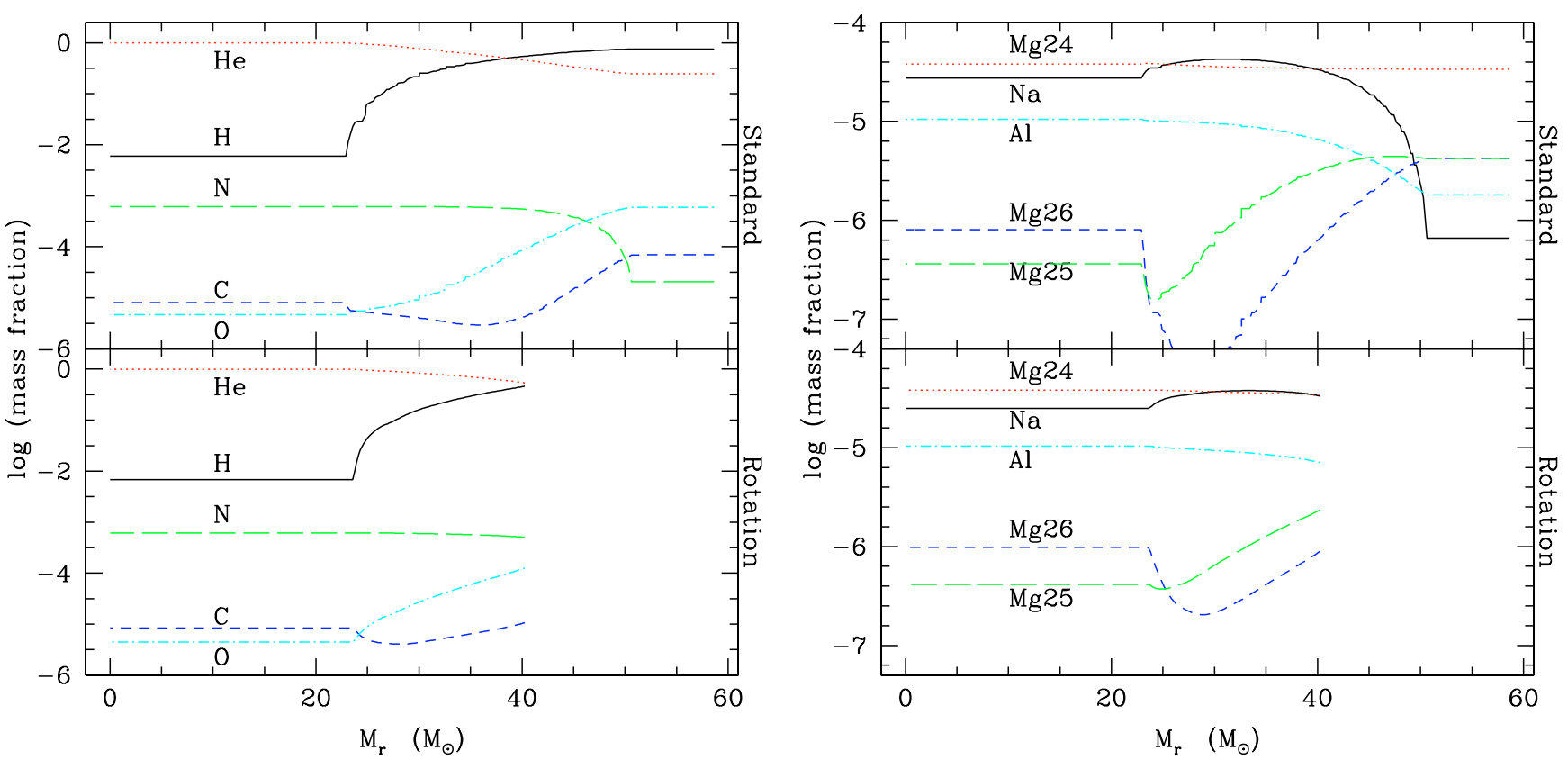

Fig. 5. Abundance profiles at the end of main sequence $\left(X_{\mathrm{c}} \simeq 0.01\right)$ for the $60 M_{\odot}$ models in the standard case $($ model $60 \mathrm{sC}$, top $)$ and for $V_{\text {ini }}=$ $800 \mathrm{~km} \mathrm{~s}^{-1}$ (model 60rC, bottom).

to mass loss which is strongly enhanced in the rotating model. The induced strong "peeling" exposes the nuclear regions. As a consequence the surface abundances and wind at the end of the main sequence show the signatures of H-processing.

\subsection{Composition of the ejecta}

During the evolution of our $60 M_{\odot}$ rotating models we can distinguish the following phases:

1. The beginning of the main sequence during which the surface velocity approaches the critical limit (between $X_{\mathrm{c}} \sim$ 0.76 to 0.65 in Fig. 4).

2. The rest of the main sequence when the surface velocity is at (or very near) the critical limit (for $X_{\mathrm{c}}$ below 0.65 in Fig. 4).

3. The beginning of the core He-burning phase when the stellar luminosity is close to the Eddington value and the surface velocity stays close to the $\Omega \Gamma$-Limit.

4. The end of the He-burning phase. From this moment on the star is away from the $\Omega \Gamma$-Limit and the mass loss is mainly radiatively-driven.

During the phases 2 and 3 described above, mass loss is mainly mechanically-driven and the stellar winds are supposed to be slow; they are enriched in H-burning products only. When the phase 4 starts, the stellar surface and the ejecta are enriched in both H-burning and He-burning products and the winds are fast.

Figure 6 presents the evolution of the surface abundances in the rotating $60 M_{\odot}$ models as a function of the remaining stellar mass from the zero age main sequence up to the end of central He-burning; the different lines correspond to models computed with the various sets of nuclear reactions described in Sect. 2.1 (60rA, B, C and D). We also indicate the range of abundances exhibited by the low-mass stars in NGC 6752 (shaded boxes).

The surface abundance variations do mimic the central ones (see Fig. 3 and the discussion in Sect. 3) with some delay due to non-instantaneous rotational mixing. In all the cases the surface abundances of carbon and oxygen at the end of the main sequence fall respectively by 0.9 and 1 dex with respect to their

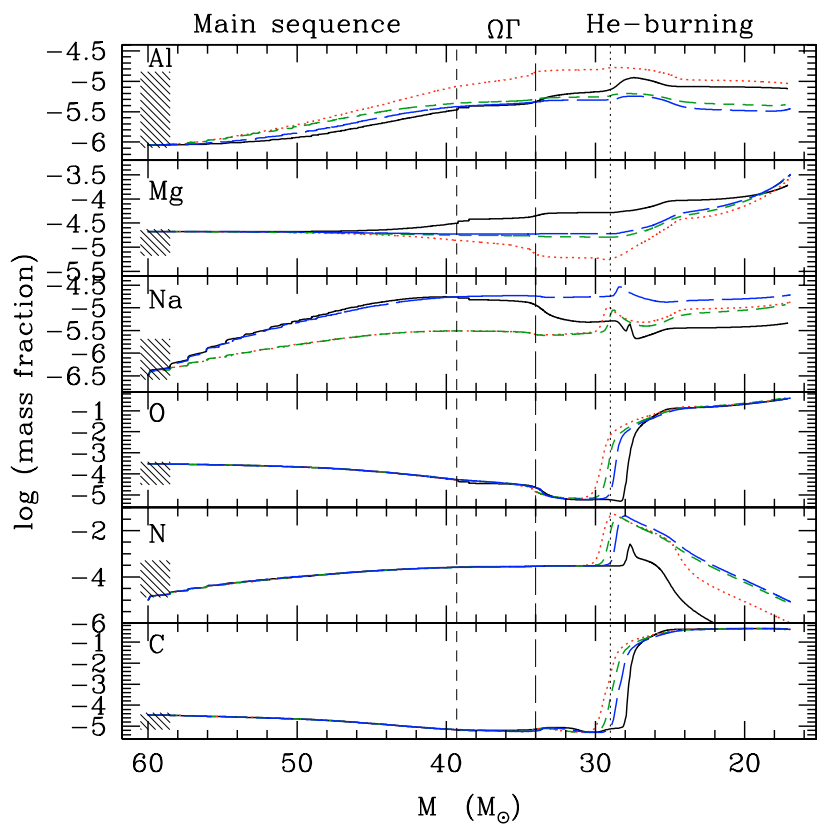

Fig. 6. Evolution of the surface abundances as a function of remaining mass for the $60 M_{\odot}$ rotating models computed with various sets of nuclear reactions: set A (full line), set B (long dashed lines), set C (short dashed lines) and set D (dotted lines). The short-dashed vertical line indicates the end of the main sequence. The $\Omega \Gamma$-Limit phase is located between the short- and long-dashed lines. The dotted line marks the moment when He-burning products start to appear at the surface. The shaded boxes on the left indicate the amplitudes of the abundance variations observed at the surface of low-mass stars in NGC 6752.

original values, whereas that of nitrogen increases by 1.5 dex. The sodium surface abundance increases by 0.8 or $1.6 \mathrm{dex}$ depending on the assumed nuclear reaction rates. Aluminum increases in all the cases but in a more efficient way when one enforces ${ }^{24} \mathrm{Mg}$-burning by increasing significantly the corresponding reaction rate (set D). As discussed previously, the 
Table 5. The main features of our models: initial mass, initial surface velocity, and for each evolutionary phase the remaining mass and the mean mass fraction in the wind for several isotopes integrated from the zero age main sequence. The 20,40 and $120 M_{\odot}$ models are computed with the reaction rates from set $\mathrm{C}$. For rotating models the last division indicates abundance when He-burning products appear at the surface, or at the end of He-burning phase depending on the first case reached.

\begin{tabular}{|c|c|c|c|c|c|c|c|c|c|c|c|c|c|}
\hline 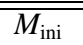 & $\overline{\overline{V_{\text {ini }}}}$ & $\overline{\bar{M}}$ & $\overline{\mathrm{H} 1}$ & $\overline{\mathrm{He} 4}$ & $\overline{\mathrm{C} 12}$ & $\overline{\mathrm{N} 14}$ & $\overline{\mathrm{O} 16}$ & $\overline{\text { F19 }}$ & $\mathrm{Na23}$ & Mg24 & $\mathrm{Mg} 25$ & Mg26 & $\overline{\mathrm{A} 127}$ \\
\hline$M_{\odot}$ & $\mathrm{km} \mathrm{s}^{-}$ & $M_{\odot}$ & \multicolumn{11}{|c|}{ Mean mass fraction in winds } \\
\hline & & & & & & STANDA & D MODE & & & & & & \\
\hline & & & & & & ind of & ral H-bu & ing & & & & & \\
\hline 20 & 0 & 19.9 & $7.5 e-1$ & $2.4 \mathrm{e}-1$ & $3.5 e-5$ & $1.0 \mathrm{e}-5$ & $3.0 e-4$ & $1.5 \mathrm{e}-8$ & $3.3 e-7$ & $1.7 e-5$ & $2.1 \mathrm{e}-6$ & $2.1 \mathrm{e}-6$ & $9.0 \mathrm{e}-7$ \\
\hline 40 & 0 & 39.7 & $75 e_{-1}$ & $2.4 \mathrm{e}-1$ & $3.5 e-5$ & $1.0 \mathrm{e}-5$ & $3.0 \mathrm{e}-4$ & $1.5 \mathrm{e}-8$ & $3.3 e-7$ & $1.7 e-5$ & $2.1 e-6$ & $e-6$ & $9.0 \mathrm{e}-7$ \\
\hline 60 & 0 & 59.2 & $5 e-1$ & $2.4 \mathrm{e}-1$ & $.5 e-5$ & $1.0 \mathrm{e}-5$ & $3.0 \mathrm{e}-4$ & $1.5 \mathrm{e}-8$ & $3.3 e-7$ & $1.7 e-5$ & $2.1 e-6$ & $2.1 \mathrm{e}-6$ & $9.0 \mathrm{e}-7$ \\
\hline 120 & 0 & 117.0 & $.5 e-1$ & $2.4 \mathrm{e}-1$ & $.5 e-5$ & $1.0 \mathrm{e}-5$ & $3.0 \mathrm{e}-4$ & $1.5 \mathrm{e}-8$ & $3.3 e-7$ & $1.7 \mathrm{e}-5$ & $2.1 \mathrm{e}-6$ & $2.1 \mathrm{e}-6$ & $9.0 \mathrm{e}-7$ \\
\hline \multicolumn{14}{|c|}{ End of central He-burning } \\
\hline 20 & 0 & 19.9 & $7.5 \mathrm{e}-1$ & $2.4 \mathrm{e}-1$ & $3.5 \mathrm{e}-5$ & $1.0 \mathrm{e}-5$ & $3.0 \mathrm{e}-4$ & $1.5 \mathrm{e}-8$ & $3.3 e-7$ & $1.7 \mathrm{e}-5$ & $2.1 \mathrm{e}-6$ & $2.1 \mathrm{e}-6$ & $9.0 \mathrm{e}-7$ \\
\hline 40 & 0 & 39.1 & $5 e-1$ & $2.5 \mathrm{e}-1$ & $3.5 e-5$ & $1.0 \mathrm{e}-5$ & $3.0 \mathrm{e}-4$ & $1.5 \mathrm{e}-8$ & $3.3 e-7$ & $1.7 \mathrm{e}-5$ & $2.1 e-6$ & $.1 \mathrm{e}-6$ & $9.0 \mathrm{e}-7$ \\
\hline 60 & 0 & 45.9 & $6.9-1$ & $3.1 \mathrm{e}-1$ & $2.6 e-5$ & $7.9 e-5$ & $2.3 e-4$ & $1.2 \mathrm{e}-8$ & $1.2 \mathrm{e}-6$ & $1.7 e-5$ & $1.6 e-6$ & $1.7 e-6$ & $1.8 \mathrm{e}-6$ \\
\hline 120 & 0 & 89.3 & $9 \mathrm{e}-1$ & $4.1 \mathrm{e}-1$ & $1.5 \mathrm{e}-5$ & $1.7 \mathrm{e}-4$ & $1.4 \mathrm{e}-4$ & $6.3 e-9$ & $2.0 \mathrm{e}-6$ & $1.7 \mathrm{e}-5$ & $9.9 \mathrm{e}-7$ & $1.2 \mathrm{e}-6$ & $3.0 \mathrm{e}-6$ \\
\hline \multicolumn{14}{|c|}{$\begin{array}{l}\text { ROTATING MODELS } \\
\text { Break-up reached }\end{array}$} \\
\hline 20 & 600 & 20.0 & $7.5 \mathrm{e}-1$ & $2.4 \mathrm{e}-1$ & $1.1 \mathrm{e}-5$ & $4.9 \mathrm{e}-5$ & $2.9 \mathrm{e}-4$ & $1.5 \mathrm{e}-8$ & $7.5 \mathrm{e}-7$ & $1.7 \mathrm{e}-5$ & $2.1 \mathrm{e}-6$ & $2.2 \mathrm{e}-6$ & $9.3 e-7$ \\
\hline 40 & 800 & 40.0 & $7.5 e-1$ & $2.4 \mathrm{e}-1$ & $3.5 e-5$ & $1.3 e-5$ & $3.0 e-4$ & $1.6 \mathrm{e}-8$ & $3.7 e-7$ & $1.7 e-5$ & $2.1 \mathrm{e}-6$ & $2.2 \mathrm{e}-6$ & $9.2 \mathrm{e}-7$ \\
\hline 60rE & 600 & 59.7 & $7.5 e-1$ & $2.5 \mathrm{e}-1$ & $3.3 e-5$ & $1.7 \mathrm{e}-5$ & $3.0 e-4$ & $1.5 \mathrm{e}-8$ & $4.2 \mathrm{e}-7$ & $1.7 e-5$ & $2.1 \mathrm{e}-6$ & $2.1 \mathrm{e}-6$ & $9.2 \mathrm{e}-7$ \\
\hline 60rA & 800 & 59.9 & $7.5 \mathrm{e}-1$ & $2.4 \mathrm{e}-1$ & $3.5 e-5$ & $1.1 \mathrm{e}-5$ & $3.0 \mathrm{e}-4$ & $1.6 \mathrm{e}-8$ & $3.5 e-7$ & $1.7 e-5$ & $2.1 \mathrm{e}-6$ & $2.1 \mathrm{e}-6$ & $9.2 \mathrm{e}-7$ \\
\hline 60rB & 800 & 59.9 & $7.5 e-1$ & $2.5 \mathrm{e}-1$ & $3.5 e-5$ & $1.1 \mathrm{e}-5$ & $3.0 \mathrm{e}-4$ & $1.6 \mathrm{e}-8$ & $3.5 e-7$ & $1.7 \mathrm{e}-5$ & $2.1 \mathrm{e}-6$ & $2.2 \mathrm{e}-6$ & $9.2 \mathrm{e}-7$ \\
\hline $60 \mathrm{rC}$ & 800 & 59.9 & $7.5 e-1$ & $2.4 \mathrm{e}-1$ & $3.5 e-5$ & $1.1 \mathrm{e}-5$ & $3.0 \mathrm{e}-4$ & $1.6 \mathrm{e}-8$ & $3.4 \mathrm{e}-7$ & $1.7 e-5$ & $2.1 \mathrm{e}-6$ & $e-6$ & $9.2 \mathrm{e}-7$ \\
\hline 60rD & 800 & 59.9 & $5 e-1$ & $2.5 \mathrm{e}-1$ & $3.5 e-5$ & $1.1 \mathrm{e}-5$ & $3.0 \mathrm{e}-4$ & $1.6 \mathrm{e}-8$ & $3.4 \mathrm{e}-7$ & $1.7 e-5$ & -6 & -6 & $2 e-7$ \\
\hline 120 & 800 & 119.5 & $7.5 e-1$ & $2.4 \mathrm{e}-1$ & $3.5 e-5$ & $1.1 \mathrm{e}-5$ & $3.0 \mathrm{e}-4$ & $1.6 \mathrm{e}-8$ & $3.4 \mathrm{e}-7$ & $1.7 e-5$ & $2.1 e-6$ & $2.1 e-6$ & $9.2 \mathrm{e}-7$ \\
\hline \multicolumn{14}{|c|}{ End of central H-burning } \\
\hline 20 & 600 & 18.4 & $e-1$ & $2.7 \mathrm{e}-1$ & $1.6 e-6$ & $8.8 \mathrm{e}-5$ & $2.5 \mathrm{e}-4$ & $1.3 \mathrm{e}-8$ & 1.5 & $1.7 e-5$ & -6 & -6 & $1.3 \mathrm{e}-6$ \\
\hline 40 & 800 & 28.8 & $2 e-1$ & $2.8 \mathrm{e}-1$ & $1.3 e-5$ & 9.36 & $2.3 e-4$ & $1.2 \mathrm{e}-8$ & 1.4 & 1.7 & -6 & -6 & -6 \\
\hline 60rE & 600 & 42.7 & $6.7 e-1$ & $3.3 e-1$ & $1.9 \mathrm{e}-5$ & $1.1 \mathrm{e}-4$ & $2.1 \mathrm{e}-4$ & $1.0 \mathrm{e}-8$ & $1.5 \mathrm{e}-6$ & $1.7 e-5$ & $1.5 \mathrm{e}-6$ & $1.6 \mathrm{e}-6$ & $2.0 \mathrm{e}-6$ \\
\hline 60rA & 800 & 39.9 & $6.8 \mathrm{e}-1$ & $3.2 \mathrm{e}-1$ & $1.9 e-5$ & $1.2 \mathrm{e}-4$ & $2.0 \mathrm{e}-4$ & $1.0 \mathrm{e}-8$ & $7.1 \mathrm{e}-6$ & $1.9 \mathrm{e}-5$ & $1.4 \mathrm{e}-6$ & $2.2 \mathrm{e}-6$ & $1.5 \mathrm{e}-6$ \\
\hline 60rB & 800 & 39.9 & $6.8 \mathrm{e}-1$ & $3.2 \mathrm{e}-1$ & $1.9 \mathrm{e}-5$ & $1.2 \mathrm{e}-4$ & $2.0 \mathrm{e}-4$ & $1.0 \mathrm{e}-8$ & $6.4 \mathrm{e}-6$ & $1.7 \mathrm{e}-5$ & $1.4 \mathrm{e}-6$ & $2.0 \mathrm{e}-6$ & $1.7 \mathrm{e}-6$ \\
\hline $60 \mathrm{rC}$ & 800 & 36 & $6.8 \mathrm{e}-1$ & $3.2 \mathrm{e}-1$ & $1.9 \mathrm{e}-5$ & $1.2 \mathrm{e}-4$ & $2.0 \mathrm{e}-4$ & $1.0 \mathrm{e}-8$ & $1.7 \mathrm{e}-6$ & $1.7 e-5$ & $1.4 \mathrm{e}-6$ & $1.6 e-6$ & $2.1 \mathrm{e}-6$ \\
\hline 60rD & 800 & 39.9 & $6.8 \mathrm{e}-1$ & $3.2 \mathrm{e}-1$ & $1.9 \mathrm{e}-5$ & $1.2 \mathrm{e}-4$ & $2.0 \mathrm{e}-4$ & $1.0 \mathrm{e}-8$ & $1.7 e-6$ & $1.6 e-5$ & $1.5 e-6$ & $1.8 \mathrm{e}-6$ & $2.7 e-6$ \\
\hline 120 & 800 & 76.6 & $6.1 \mathrm{e}-1$ & $3.9 \mathrm{e}-1$ & $1.7 \mathrm{e}-5$ & $1.8 \mathrm{e}-4$ & $1.3 \mathrm{e}-4$ & $6.3 \mathrm{e}-9$ & $2.2 \mathrm{e}-6$ & $1.7 \mathrm{e}-5$ & $9.2 \mathrm{e}-7$ & $1.1 \mathrm{e}-6$ & $3.1 \mathrm{e}-6$ \\
\hline \multicolumn{14}{|c|}{ Appearence of He-burning products at the surface/End of central He-burning } \\
\hline 20 & 600 & 16.4 & $7.0 \mathrm{e}-1$ & $3.0 \mathrm{e}-1$ & $1.6 \mathrm{e}-6$ & $1.1 \mathrm{e}-4$ & $2.3 e-4$ & $1.1 \mathrm{e}-8$ & $1.8 \mathrm{e}-6$ & $1.7 e-5$ & $1.7 \mathrm{e}-6$ & $1.9 \mathrm{e}-6$ & $1.6 e-6$ \\
\hline 40 & 800 & 18.5 & $5.7 \mathrm{e}-1$ & $4.3 \mathrm{e}-1$ & $9.3 e-6$ & $1.6 \mathrm{e}-4$ & $1.6 \mathrm{e}-4$ & $8.0 \mathrm{e}-9$ & $2.1 \mathrm{e}-6$ & $1.7 e-5$ & $1.2 \mathrm{e}-6$ & $1.3 e-6$ & $2.7 \mathrm{e}-6$ \\
\hline 60rE & 600 & 33.2 & $5.2 \mathrm{e}-1$ & $4.8 \mathrm{e}-1$ & $1.4 \mathrm{e}-5$ & $1.7 \mathrm{e}-4$ & $1.4 \mathrm{e}-4$ & $7.2 \mathrm{e}-9$ & $2.0 \mathrm{e}-6$ & $1.7 e-5$ & $1.1 \mathrm{e}-6$ & $1.2 \mathrm{e}-6$ & $3.0 \mathrm{e}-6$ \\
\hline 60rA & 800 & & $5.4 \mathrm{e}-1$ & $4.6 e-1$ & $1.5 e-5$ & $1.7 \mathrm{e}-4$ & $1.5 \mathrm{e}-4$ & $7.2 \mathrm{e}-9$ & $8.6 e-6$ & & $1.3 e-6$ & $2.0 \mathrm{e}-6$ & $2.5 \mathrm{e}-6$ \\
\hline 60rB & 800 & & $5.5 e-1$ & $4.5 \mathrm{e}-1$ & $1.5 \mathrm{e}-5$ & $1.7 \mathrm{e}-4$ & $1.5 \mathrm{e}-4$ & $7.2 \mathrm{e}-9$ & $1.0 \mathrm{e}$ & $1.7 e-5$ & $1.1 \mathrm{e}-6$ & $1.5 \mathrm{e}-6$ & $2.6 e-6$ \\
\hline 60rC & 800 & 30.7 & $5.5 \mathrm{e}-1$ & $4.5 \mathrm{e}-1$ & $1.5 \mathrm{e}-5$ & $1.7 \mathrm{e}-4$ & $1.5 \mathrm{e}-4$ & $7.3 e-9$ & 2.0 & $1.7 \mathrm{e}$ & $1.1 \mathrm{e}-6$ & $1.2 \mathrm{e}-6$ & $3.0 \mathrm{e}-6$ \\
\hline 60rD & 800 & & e- -1 & $4.5 \mathrm{e}-$ & $1.5 \mathrm{e}-5$ & $1.7 \mathrm{e}-4$ & $1.5 \mathrm{e}-4$ & $7.3 e-9$ & 2.0 & $1.4 \mathrm{e}-5$ & $1.1 \mathrm{e}-6$ & $1.6 \mathrm{e}-6$ & $5.5 e-6$ \\
\hline 120 & 800 & 68.7 & $5.0 \mathrm{e}-1$ & $5.0 \mathrm{e}-1$ & $1.5 \mathrm{e}-5$ & $2.0 \mathrm{e}-4$ & $1.1 \mathrm{e}-4$ & $5.2 \mathrm{e}-9$ & $2.2 \mathrm{e}-6$ & $1.7 e-5$ & $8.4 \mathrm{e}-7$ & $9.6 \mathrm{e}-7$ & $3.5 \mathrm{e}-6$ \\
\hline
\end{tabular}

$\mathrm{O}-\mathrm{Na}$ anticorrelation appears in all the cases. However an increase of the ${ }^{24} \mathrm{Mg}(\mathrm{p}, \gamma)$ reaction rate is needed to correctly produce the $\mathrm{Mg}-\mathrm{Al}$ anticorrelation.

The H-burning signatures are reinforced at the stellar surface at the beginning of the central He-burning phase mainly as a result of previous mass loss episodes.

However, later on the products of He-burning appear at the surface. In particular the oxygen abundance rises steeply from $10^{-5}$ to nearly 0.6 in mass fraction when the total stellar mass is lower than $\sim 28 M_{\odot}$. C and $\mathrm{N}$ also display strong enhancement at this phase. After that, the abundance of nitrogen decreases transformed into ${ }^{22} \mathrm{Ne}$.

Thus, the ejecta of fast rotating massive stars display the chemical patterns observed in GC stars both on the main sequence and during the $\Omega \Gamma$-Limit phase. These are exactly the phases where matter is ejected by gently blowing winds which can be easily retained within the GC potential well. The He-burning products are released in the interstellar matter through fast radiatively-driven winds.

\subsection{Effect of the initial rotation velocity}

In order to investigate the impact of the initial rotation velocity on the overall predictions we have computed a $60 M_{\odot}$ model with an initial velocity of $600 \mathrm{~km} \mathrm{~s}^{-1}$ (model 60rE, with set C) instead of $800 \mathrm{~km} \mathrm{~s}^{-1}$ (model $60 \mathrm{rC}$ ). The mixing of chemicals is less efficient in the slowest model as the shear turbulence, which dominates the transport processes, decreases with lower angular velocity. However, model 60rE reaches the break-up velocity later during the main sequence (when the central hydrogen mass fraction is 0.4 instead of 0.65 in model $60 \mathrm{rC}$ ). Both effects tend to compensate each other: the mixing has a lower efficiency but has more time to act before the star reaches the break-up. This leads to abundance variations that are even more pronounced. As an example the nitrogen abundance in winds when break-up is reached is 55\% higher in model 60rE than in model 60rC (see Table 5).

When the stars are at break-up, the mass loss rate is higher for a lower initial rotation velocity: compared to the standard (non-rotating) case it is 29 and 24 times higher in models 60rE 
and $60 \mathrm{rC}$ respectively. The total stellar mass at the end of the main sequence is higher (by $\sim 3 M_{\odot}$ ) in model $60 \mathrm{rC}$. These differences play only a minor role in the composition of the yields which differ by only a few percent between the two models.

With an initial velocity around $300 \mathrm{~km} \mathrm{~s}^{-1}$ the $60 M_{\odot}$ star would fail to reach the break-up and the conditions for the WFRMS scenario would not be fulfilled. If we infer models with various initial mass and metallicity (Ekström et al., in preparation), the $60 M_{\odot}$ would reach the critical velocity with an initial velocity as low as $400 \mathrm{~km} \mathrm{~s}^{-1}$. However fast rotation is possibly a characteristic of massive stars in a very dense environment such as that forming GC where multiplicity and stellar collisions can play an important role. This point is discussed in more detail in Sect. 7.

\subsection{Summary}

The WFRMS appears to be very promising. Rotational mixing efficiently transports elements from the convective core to the surface. A high initial rotation velocity allows the star to reach break-up early on the main sequence and to eject important quantities of material loaded with $\mathrm{H}$-burning products. This material is probably ejected through a slow wind and is likely to remain in the GC potential well (see Sect. 7).

The abundance patterns at the stellar surface and in the ejecta follow those created in the core with some delay. The amplitude of the predicted $\mathrm{O}-\mathrm{Na}$ anticorrelation well reproduces the observations. Although the $60 M_{\odot}$ star produces too little $\mathrm{Al}$ whith the nuclear experimental value for the ${ }^{24} \mathrm{Mg}(\mathrm{p}, \gamma)$ reaction rate, an increase of this rate allows one to explain the observed $\mathrm{Mg}-\mathrm{Al}$ anticorrelation.

\section{Dependence on the initial stellar mass}

We now discuss how the theoretical predictions depend on the initial stellar mass. Standard and rotating models with initial masses ranging between 20 and $120 M_{\odot}$ are presented; in all cases we used the set $\mathrm{C}$ for the nuclear reaction rates.

\subsection{Nucleosynthesis and mixing}

The initial mass has a direct effect on nucleosynthesis through the changes in the central temperature (see Fig. 7). As a result the $\mathrm{NeNa}$ and $\mathrm{MgAl}$ chains are more active in the warmer convective core of more massive stars.

The evolution along the main sequence of the central abundances of some interesting nuclei is shown in Fig. 8 for the 20 and $120 M_{\odot}$. It can be compared to Fig. 3 . The following differences can be noted:

- The CNO equilibrium value of $\mathrm{O}$ is slightly lower in the higher temperature regime of the $120 \mathrm{M}_{\odot}$ stellar model.

- ${ }^{23} \mathrm{Na}$ is first produced to the same extent in both models, but later on it decreases faster in the more massive star.

- In the $120 M_{\odot}$ model ${ }^{24} \mathrm{Mg}$ decreases by $1.2 \mathrm{dex}$ at the end of the H-burning phase, while the $20 M_{\odot}$ model never reaches sufficiently high temperatures for this element to burn.

- ${ }^{27} \mathrm{Al}$ is produced earlier in the $120 M_{\odot}$ model. Nevertheless the plateau stays at the same level in both models. Only at the very end of the main sequence does the $\mathrm{Al}$ abundance rapidly increase in the more massive star as a result of ${ }^{24} \mathrm{Mg}$ burning.

Thus the main relevant difference between both stars concerns the $\mathrm{MgAl}$ chain. Only in the $120 M_{\odot}$ model and at the very end

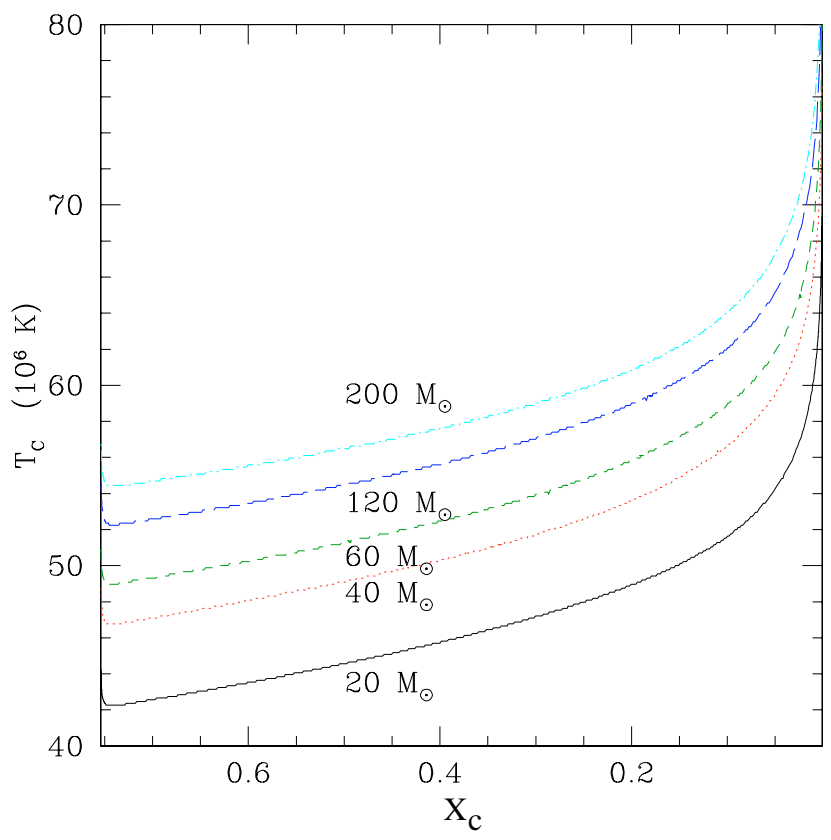

Fig. 7. Evolution of the central temperature for the 20, 40, 60, 120 and $200 M_{\odot}$ rotating stars as a function of the central hydrogen abundance during the main sequence.

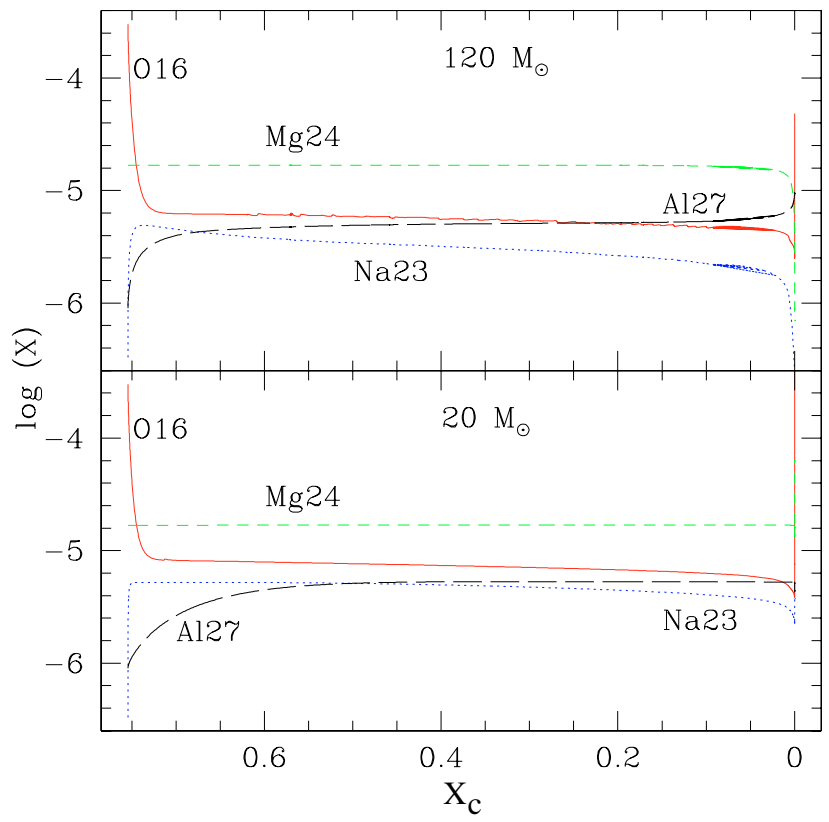

Fig. 8. Evolution of the central abundances of ${ }^{16} \mathrm{O},{ }^{23} \mathrm{Na},{ }^{24} \mathrm{Mg}$ and ${ }^{27} \mathrm{Al}$ as a function of the central hydrogen abundance on the main sequence for the 20 and $120 M_{\odot}$ rotating models.

of central H-burning are ${ }^{24} \mathrm{Mg}$ and ${ }^{27} \mathrm{Al}$ respectively destroyed and produced. Even the $200 M_{\odot}$ star does not reach a central temperature high enough to convert ${ }^{24} \mathrm{Mg}$ before the end of main sequence.

As discussed in Meynet \& Maeder (2000), rotational mixing is more efficient when the initial stellar mass is higher, favoring the transport of the nuclear products outwards. At a given evolutionary stage, more massive stars also present stronger winds, both radiatively- and mechanically-induced. 


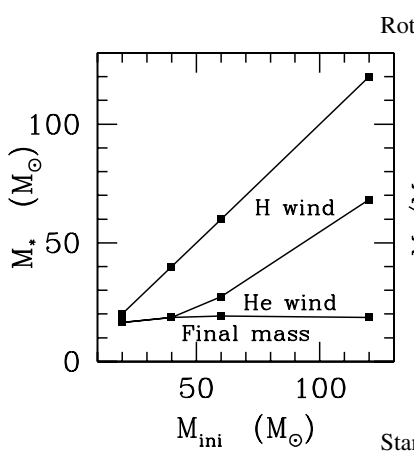

Rotating models

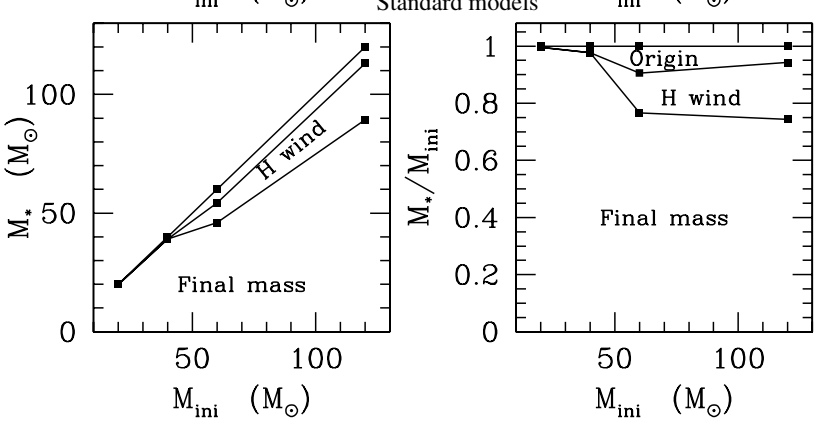

Fig. 9. Composition of the matter ejected in the winds as function of initial stellar mass for the rotating and standard models (upper and lower panels respectively). The ejecta are presented in solar mass (left) or scaled with respect to initial mass (right). The winds enriched in $\mathrm{H}-$ and He-burning products are quoted respectively as " $\mathrm{H}$ wind" and "He wind". "Origin" refers to matter which is not modified by nuclear burning. "Final mass" indicates the mass of the stars at the end of central He-burning.

\subsection{Winds}

Figure 9 illustrates the differences between the standard and rotating models for the wind composition and the remaining stellar mass at the end of central He-burning. As already discussed, the rotating models lose much more mass than the standard ones for three reasons: first, they experience mechanical winds when they reach the break-up velocity; second, their radiatively-driven winds are enhanced by a correcting factor due to rotation; third, they enter the WR phase earlier in their evolution.

The rotating models with an initial mass above $40 M_{\odot}$ lose about half of their mass through winds loaded with H-burning products. This matter is released very smoothly in the interstellar medium, with velocities low enough to remain in the GC. This is not the case of the winds of non-rotating massive stars. Indeed in that case, the wind velocities on the main sequence are of the order of 1000 to $2000 \mathrm{~km} \mathrm{~s}^{-1}$ (see e.g. Lamers et al. 1995) and this material is probably thrown out of the gravitational potential well of the cluster.

\section{Comparison with observed abundance variations in GCs}

Here we explore how the ejecta of massive rotating stars can be used to form low-mass stars displaying abundance variations in light elements. It is beyond the scope of the paper to present a detailed study of the interaction between the disk enclosing fast rotating massive stars and the surrounding protocluster gas. We will thus only make some simple assumptions to verify whether massive stars can be the progenitors of the long-lived stars we observe today.

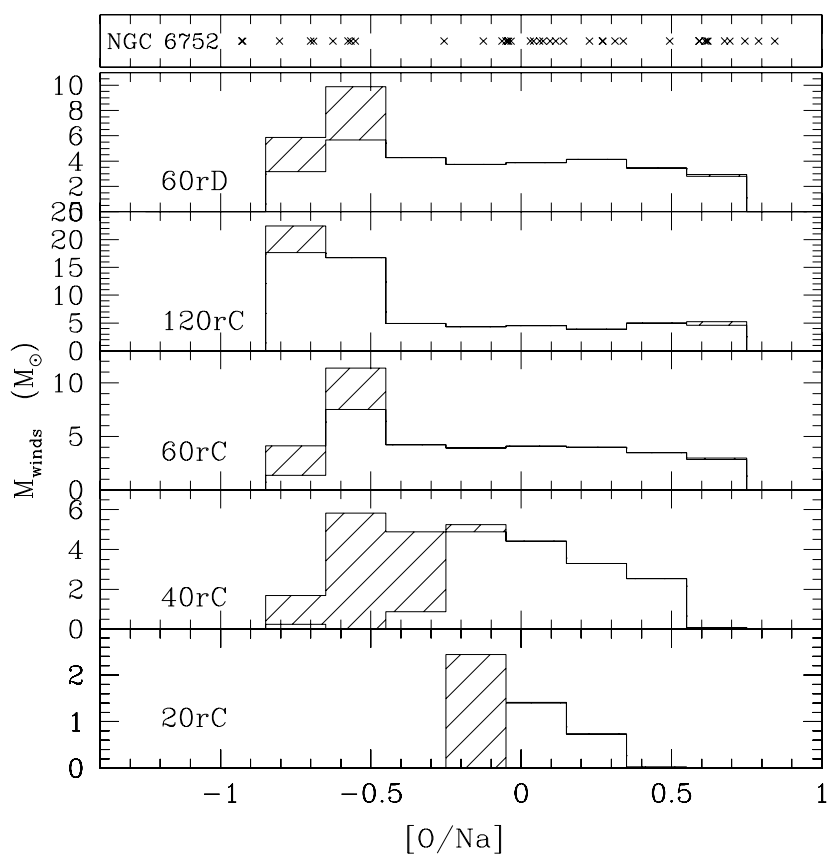

Fig. 10. (Top) Crosses are observed [O/Na] ratio in individual NGC 6752 stars computed from the data of Grundahl et al. (2002) and Yong et al. (2003). (Other panels) Theoretical histograms built from the wind composition of rotating massive stars in which $30 \%$ of pristine matter is added as required by the Li behavior (see the text). The white and shaded areas indicate respectively the slow (i.e., when the star is at breakup) and fast winds.

As the disks may be mixed with some pristine gas, we need first to evaluate the amount of this dilution. For that we use abundance variations in Li measured by Pasquini et al. (2005) in NGC 6752: $\mathrm{Li}$ is found to be as low as $A(\mathrm{Li})=1.93$ (Alonso temperature scale) in the most polluted stars. In the present models we do not explicitly follow the nucleosynthesis of lithium but we can reasonably assume that this fragile element is completely destroyed due to high temperature in the stellar interior and to the strong mixing induced by rotation. If we assume that the initial lithium abundance of the intracluster gas corresponds to the cosmological value of 2.61 (Coc et al. 2004) and that no lithium is present in the wind of massive stars, we need to add about $30 \%$ of pristine gas to those winds to obtain the extreme value of lithium (see also Prantzos \& Charbonnel, in preparation).

As a first estimate we use this dilution factor between the stellar winds and the original matter and check whether it allows us to reproduce all the observed abundance variations. In real proto-globular cluster there will be a dispersion in the amount of dilution which in turn creates a variation in the abundances of low-mass stars.

Figures 10-12 display the composition of the mixed matter formed by adding $30 \%$ of pristine gas to massive star ejecta as well the observed abundance distributions in NGC 6752 (Grundahl et al. 2002; Yong et al. 2003; Carretta et al. 2005). The theoretical histograms indicate the mass ejected by winds mixed with pristine gas resulting in a given chemical composition. The white areas correspond to the mass lost at break-up, while the hatched regions indicate that the corresponding winds are released when stellar rotation is well below its critical value (this occurs at the beginning of the main sequence and during the He-burning phase). This latter component thus has a high velocity and probably escapes the GC potential well. Only the H-rich loaded winds are shown here. The He-rich winds are released at 


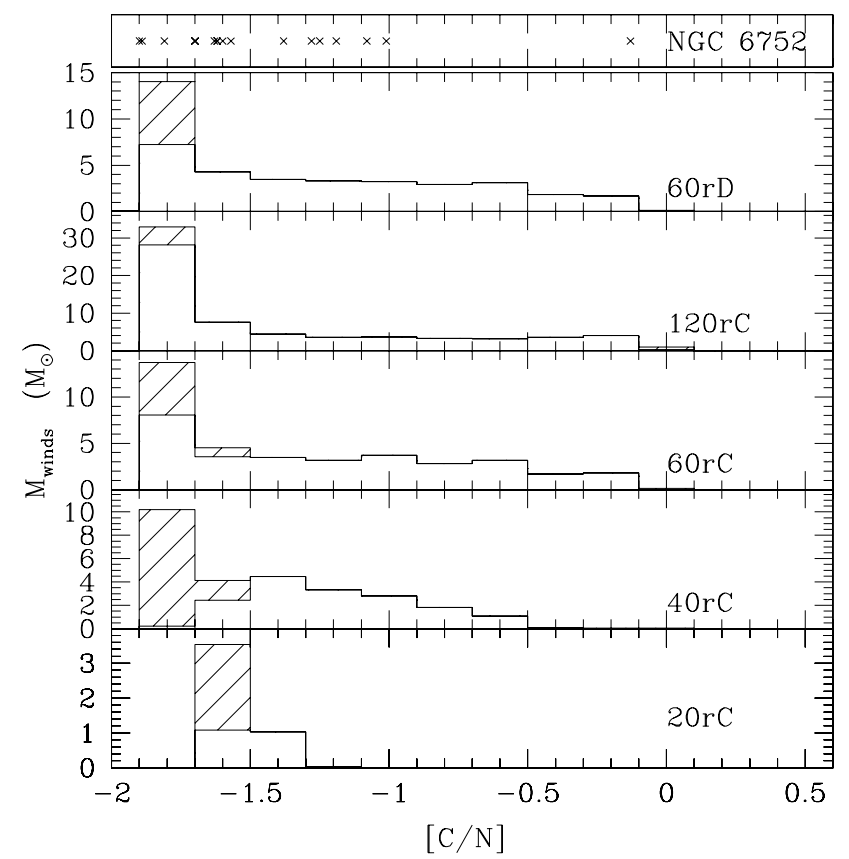

Fig. 11. Same as Fig. 10 for $[\mathrm{C} / \mathrm{N}]$ ratio (data from Carretta et al. 2005).

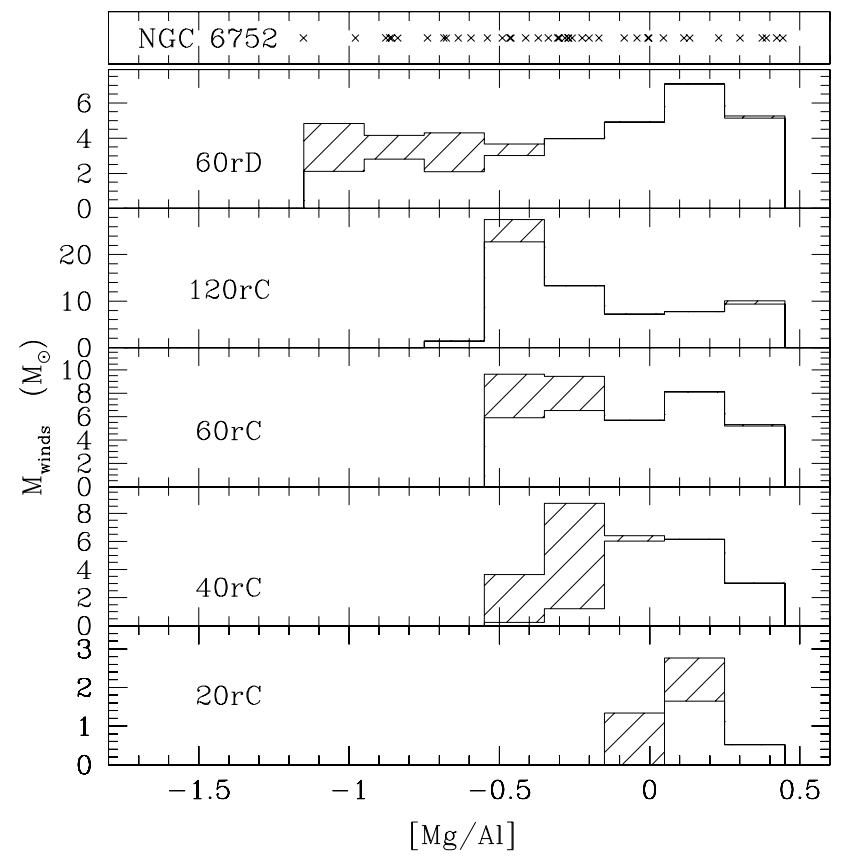

Fig. 12. Same as Fig. 10 for $[\mathrm{Mg} / \mathrm{Al}]$ ratio (data from Grundahl et al. 2002; Yong et al. 2003).

high velocity and are supposed to escape the potential well of the GC.

We first concentrate on the $[\mathrm{O} / \mathrm{Na}]$ distribution. The abundance pattern in the mixed matter of the 60 and $120 M_{\odot}$ models covers the entire observational range. The case of the less massive stars is different. At first glance the predictions for the $40 M_{\odot}$ model also account well for the observed dispersion. This is not the case when one considers only the slow winds ejected at break-up. The $20 M_{\odot}$ star follows the same trend but to a smaller extent. Let us note that the highest $[\mathrm{O} / \mathrm{Na}]$ ratio for both the 20 and $40 M_{\odot}$ stars is shifted to the left compared to more massive models. This is due to the longer time required to reach critical velocity at the beginning of the main sequence. When the

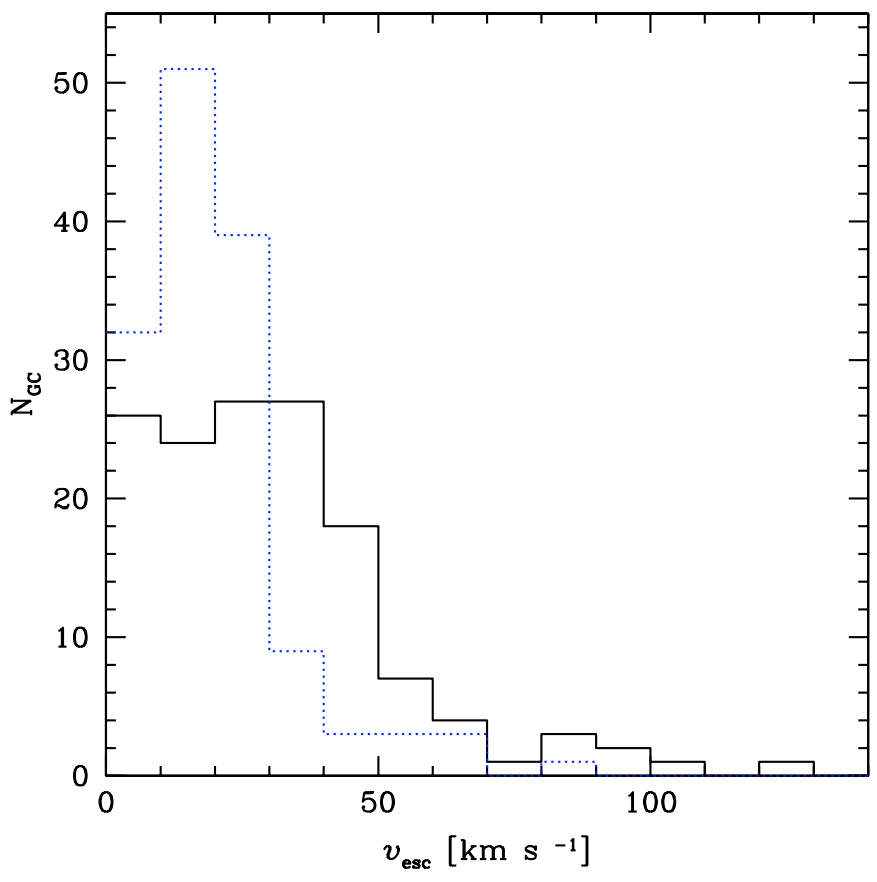

Fig. 13. Histogram of the number of globular clusters having a given value of the escape velocity. The data are taken from Gnedin et al. (2002). The continuous line shows the results for the escape velocity at the center of the cluster, the dotted line at the cluster half-mass radius.

break-up is reached, rotational mixing had more time to transport the elements through the radiative envelope.

For the $[\mathrm{C} / \mathrm{N}]$ distribution (Fig. 11) we find that the ejecta of rotating massive stars cover the observational range. Again the $20 M_{\odot}$ and $40 M_{\odot}$ models, being more mixed before reaching break-up, do not expel matter with original composition (see Table 5).

Regarding the $[\mathrm{Mg} / \mathrm{Al}]$ ratio we see that the winds of all the models computed with the set $\mathrm{C}$ (experimental limits) present lower abundance variations than required by the observed distribution. In addition magnesium isotopic ratios obtained in these models are at odds with the observed ones: even in the case of the $120 M_{\odot}$ models, aluminum is built mainly from ${ }^{25} \mathrm{Mg}$ and ${ }^{26} \mathrm{Mg}$. Only model 60rD covers the whole observational range both in terms of $\mathrm{Mg}$ and $\mathrm{Al}$ abundances and of $\mathrm{Mg}$ isotopic ratios. The same would be true for other masses computed with set D. This difficulty remains even in the extreme case where present day halo stars are naked cores of massive stars having undergone strong evaporation at the end of the main sequence. Only at the end of the main sequence do the 60 and $120 M_{\odot}$ models manage to destroy ${ }^{24} \mathrm{Mg}$ by more than 0.3 dex and to enhance ${ }^{27} \mathrm{Al}$. However at that time the high central temperature leads to a strong destruction of ${ }^{23} \mathrm{Na}$ which falls near to or below its initial value.

\section{Discussion}

Let us now discuss some details of the WFRMS scenario.

\subsection{Kinematics and topology of the ejected material}

Figure 13 shows the distribution of the globular clusters as a function of the escape velocity estimated at the center and at half-mass radius of the present-day cluster (continuous and dotted line respectively, Gnedin et al. 2002). More than $60 \%$ of the 
141 globular clusters used to build the histogram (i.e. the large majority of the known galactic globular clusters) have an escape velocity at the center between 20 and $100 \mathrm{~km} \mathrm{~s}^{-1}$. The range is shifted to lower values if escape velocities estimated at half-mass radius are used. These values imply that all the wind ejecta of O-type stars and of WR stars (wind velocities of a few thousandths of $\mathrm{km} \mathrm{s}^{-1}$ ), of Luminous Blue Variable (wind velocities of a few hundred $\mathrm{km} \mathrm{s}^{-1}$ ) as well as the supernova ejecta (velocities of the ejecta of the order of $10000 \mathrm{~km} \mathrm{~s}^{-1}$ ) will be lost by the globular cluster. However, the escape velocity of the present day globular clusters may be different from the one at the time of their formation. If the present globular clusters were the remnants of much more massive systems having lost a large part of their initial mass, then the present day escape velocity might be a poor estimate of the escape velocity at the time of their formation $^{6}$. Also, the interstellar medium at the time of globular cluster formation was much denser and thus the matter ejected was slowed down by the bow shock between the ejecta and the ambient medium as shown for instance in the numerical models by Freyer et al. (2003; see also Garcia-Segura et al. 1996). It is however difficult, without a more precise view of the initial conditions of the globular cluster formation, to arrive at a firm conclusion $^{7}$. In the following, in the absence of better knowledge of the globular cluster formation conditions, we consider that the present escape velocities are representative of the escape velocities at the time of the chemical inhomogeneities formation.

Thus we examine whether our massive star models are able to eject matter at sufficiently low velocities to allow the ejecta to remain in the potential well of the globular cluster. Rotation might help in this respect. Indeed, in situations where the surface equatorial velocity is such that the centrifugal acceleration exactly counterbalances the gravity, matter can be launched into a Keplerian orbit and an equatorial disk easily forms, as schematically shown in Fig. 14. The material mainly "mechanically" ejected into the disc will be possibly available for further star formation, unless it falls back onto the star and/or is ejected outside the globular cluster by further violent wind episodes or by the supernova explosion. Let us briefly discuss these different possibilities:

- Fall back of the disk material? Be stars are main-sequence stars surrounded by a disc (Porter \& Rivinius 2003). The origin of the disk is probably stellar rotation near the critical limit as convincingly discussed by Townsend et al. (2004). Thus these objects are the observable counterparts of the stellar models we are interested in here. Observations (see the review by Rivinius 2005) indicate that Be star disks are eroded and finally dissipate, adding their material to the ambient interstellar medium. Thus these observations would favor the loss of the disk material around Be stars and not its fall back onto the star.

- Ejection by fast stellar winds? When the star no longer rotates with the critical velocity, matter is no longer preferentially ejected in the equatorial plane, and different situations may occur. It might be that the timescale for disk dissipation is very short and thus that most of the disk material

${ }^{6}$ More precisely we need the escape velocity at the time of the chemical inhomogeneities formation, which in our present model would be different from the time of formation of the first stellar generation.

7 One could argue that we can observe young globular clusters and thus obtain some constraints on their formation. However, many (if not all) young super star clusters observed today and believed to be young globular clusters result from galaxy merging or interactions. It is not clear that the old galactic globular clusters were formed in this way.

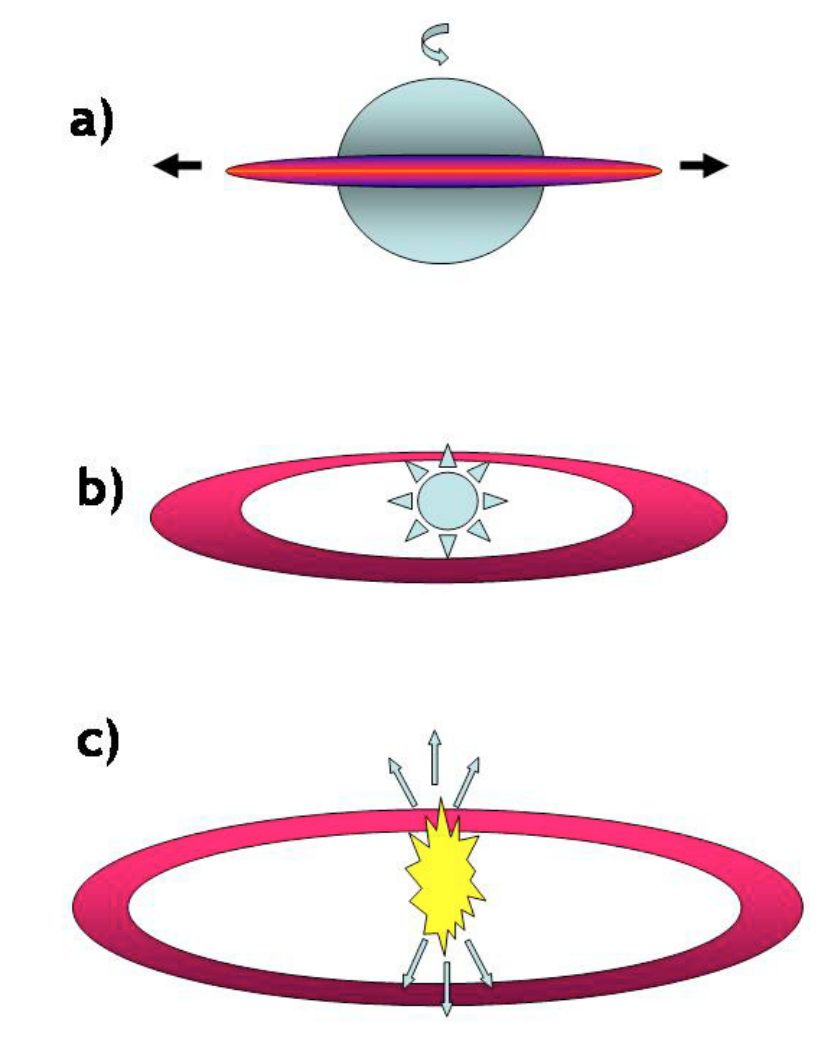

Fig. 14. Schematic view of the WFRMS scenario showing the possible geometry of the stellar ejecta at various evolutionary phases: a) during the main sequence, when the star rotates near or at the critical velocity, matter is preferentially ejected in the equatorial plane by the action of the centrifugal acceleration; b) after the main sequence, the surface velocity is no long critical and the wind is triggered mainly by radiation. It is no longer equatorial and becomes isotropic; c) the supernova explosion resulting from an initially fast spinning star, if it occurs, may favor ejection through jets aligned along the rotational axis.

has already disappeared when the faster radiatively driven winds set in. In that case there is the possibility that the fast wind pushes this freshly ejected material out of the globular cluster, which will be then lost. The most favorable case for keeping the material inside the globular cluster is that the disk has a sufficiently long lifetime to still keep most of its material when the fast winds set in. In that case, if the star is still in the blue part of the HR diagram (as is the case for our 60 and $120 M_{\odot}$ ) and if its rotation is still sufficiently close to the critical value, the wind will be preferentially ejected along the rotational axis. This would thus prevent the winds from sweeping off the disk. If the star has a rotational velocity well below the critical one, the wind is isotropic and that the fraction of the wind which may interact with the disk will likely be too small to destroy it (see Fig. 14).

- Will the disk be ejected by the supernova explosion? If a black hole is formed that swallows all the mass of the pre-supernova, no explosion occurs and the disk will not be affected. If, on the other hand, a supernova explosion occurs, the fast rotation of the core may favor ejection along the rotational axis as in the models of Maeda \& Nomoto (2003). In that case there is some chance that the disk will not be affected by the supernova explosion, as illustrated schematically in Fig. 14. The disk material can also be destroyed and ejected out of the GC and pushed away by the winds and/or supernova shocks from nearby massive stars. Our scenario will then be possible only if on relatively short 


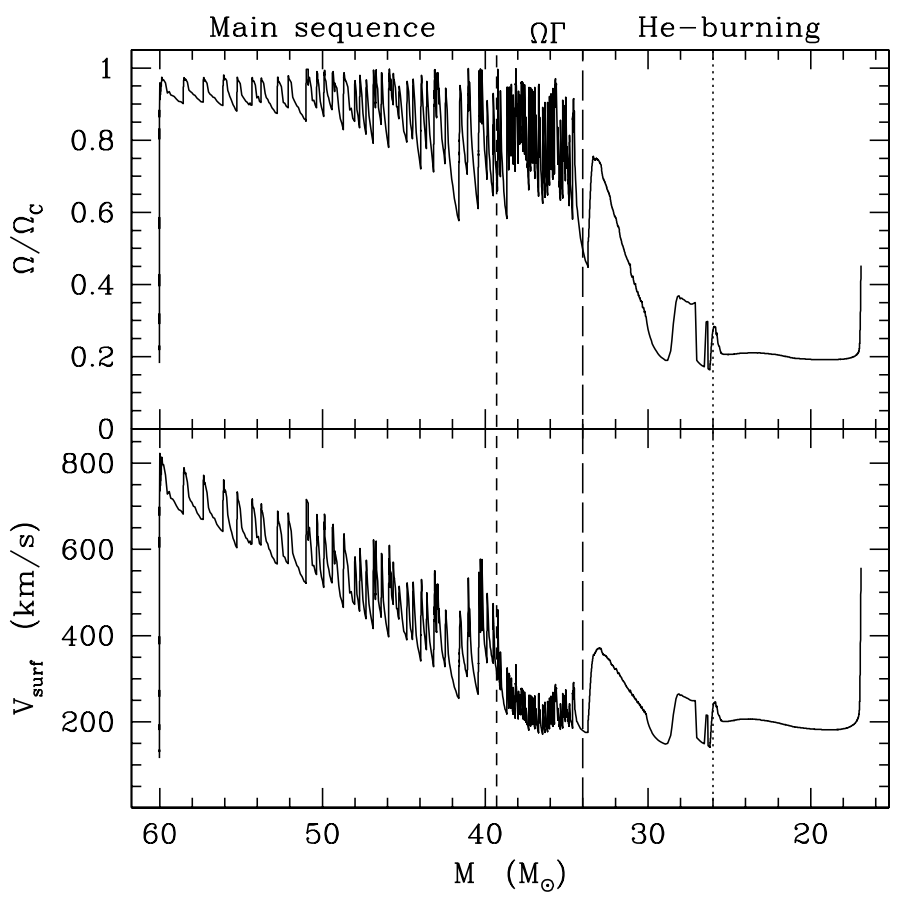

Fig. 15. Evolution of the ratio between the surface angular velocity and the classical critical velocity (top) and of the surface equatorial velocity (bottom) as a function of the remaining mass for the $60 M_{\odot}$ star. Vertical short-dashed line corresponds to the end of the main sequence and dotted line indicates the moment when He-burning products show up at the surface. The $\Omega \Gamma$-Limit phase is delimited by the short- and long-dashed lines.

timescales (shorter than the average time between two supernova events), new stars form in the disk, or more probably in the interaction region between the disk material and the interstellar medium. In that case, the fast moving material will escape following the nearly empty channels left behind by the star formation process, in line with the scenario proposed by PC06.

These ideas are highly speculative and will have to be checked by detailed hydrodynamic calculations. For the purpose of the present discussion, we suppose that the equatorial disk that formed when the star reached the critical limit consists of material that will be made available for forming new stars in the globular cluster.

Now, in the framework of the above hypothesis, we examine when this occurs during the evolution of our stellar models and whether sufficient mass with the adequate chemical composition is ejected during these phases. The present models show that the critical limit can be reached at two epochs:

1. During the main sequence, provided that the initial velocity is high enough. From Fig. 15 one sees that our $60 M_{\odot}$ model with $v_{\text {ini }}=800 \mathrm{~km} \mathrm{~s}^{-1}$ remains near the break-up limit during nearly the whole main sequence. At the turnoff, the star has lost more than $20 M_{\odot}$.

2. After the main sequence we saw that stars massive enough do reach the $\Omega \Gamma$-limit which maintains them at the break-up ${ }^{8}$ due to the increase in luminosity. Strong mass loss ensues: During this phase the $60 M_{\odot}$ star expels more than $5 M_{\odot}$ (see Fig. 15). We suppose here that this material will still be

8 At this stage, the critical velocity is lowered with respect to its classical expression (see Maeder \& Meynet 2000). preferentially ejected along the equatorial plane and will join the disk of slowly outflowing material.

\subsection{Composition of the ejected material}

In the previous sections we have seen that the chemical composition of the ejected material presents many similarities with the abundance patterns observed at the surface of globular cluster stars. If one focuses on the matter ejected during the main sequence, one sees however that the theoretical variations can account only partly for the observed range in oxygen and sodium. However, slightly later in the evolution, strongly CNO processed material is ejected and the most extreme observational cases can be reproduced (see Table 5). The observed variations of $\mathrm{Mg}$ and $\mathrm{Al}$ can also be reproduced, provided the rate of the ${ }^{24} \mathrm{Mg}(p, \gamma)$ reaction is enhanced by about a factor of 1000 for temperatures around $50 \times 10^{6} \mathrm{~K}$. However if the non-enhanced rate for this reaction is correct, then our models reproduce only part of the $\mathrm{Mg}-\mathrm{Al}$ anticorrelation.

Fast rotating massive stars are extremely good candidates to provide the material from which the long-lived low-mass stars that we are presently observing formed. The process leading to the incorporation of this material in new stars is not known, but we have given some reasons to believe that the ejecta can be retained in the globular cluster. The stars forming from the wind ejecta of massive stars mixed with some pristine interstellar gas would also present higher helium abundances (Salaris et al. 2006) and of course high $\mathrm{C} / \mathrm{N}$ ratios. The values of the ${ }^{12} \mathrm{C} /{ }^{13} \mathrm{C}$ would be quite low: all the massive stars have ratios below 5 . The corresponding implications will be studied in a subsequent work.

\subsection{Why only in GCs?}

The environment of field halo stars is very different from the one of globular clusters. However, in the past, they probably shared, at least for a short time, a cluster environment. These clusters were probably much less massive and/or much less concentrated than the progenitors of the present day globular clusters. This is required for these clusters to have evaporated, being completely disrupted either by the energy injections of the first supernovae ${ }^{9}$, or by tidal effects. In that case, star formation triggered by the wind/SN shocks from evolving massive stars might have been quenched, rendering impossible the birth of field stars with processed material.

Another possibility, also related to the high stellar density in GCs, could be that such an environment favors higher rotational velocities than less dense environments. It is however not obvious that denser environments would favor high rotational velocities, the stellar encounters being able to both spin up the stars or slow them down. Some observations find higher rotational velocities in clusters than in the field: Huang \& Gies (2006) determined the projected rotational velocities of 496 OB stars in clusters within the approximate age range 6-73 Myr. They found that there are fewer slow rotators among the cluster B-type stars relative to nearby B stars in the field. Strom et al. (2005) also found that stars in $h$ and $\chi$ Persei tend to rotate faster than the field star counterparts. A similar conclusion reached by Dufton et al. (2006) on the basis of the rotational velocities of stars in the two clusters NGC 3293 and NGC 4755. We cannot draw

\footnotetext{
9 The gravitational energy of a globular cluster is of the order of $10^{51} \mathrm{erg}$ i.e. of the same order of magnitude as the kinetic energy emitted by a core collapse supernova explosion.
} 
firm conclusions about the origin of this difference, and these observations may support the view that stars born in dense environments may have faster rotational velocities than stars born in loose aggregates.

An alternative to this view is to consider that all the field stars were born in the progenitors of the present day globular clusters. Field halo stars contain at least a hundred times the mass of globular clusters (Woltjer 1975). The above scenario would therefore imply a very efficient mechanism that would remove $99 \%$ of stars initially formed in the progenitor of the present day GC. Moreover, this mechanism should either be more rapid than the enrichment of the interstellar medium by massive stars, or should only remove stars with no chemical inhomogeneities. While it appears difficult to favor such a view, it would have the advantage of making the stars presenting inhomogeneities a very small subset of the whole stellar population in a given cluster and thus alleviate the need for a very flat IMF $(\mathrm{PC} 06)^{10}$.

\section{Conclusion}

Massive stars have been discarded in the past in the context of the GC self-enrichment scenario because these objects are expected to produce iron and to have fast winds.

In the present paper, we propose the Wind of Fast Rotating Massive Star scenario in order to explain the chemical inhomogeneities observed in GC stars. The key point of this scenario is the fast rotation of massive stars. Each one of these two characteristics is important:

- Fast rotation is needed to remove material from the stellar surface and inject it with a low velocity in the interstellar medium. It triggers internal mixing which brings to the surface material processed in the core. This enables a star to eject material with chemical compositions similar to that observed at the surface of GC stars.

- Massive stars have short lifetimes and can release $\mathrm{H}$-synthesized material while low-mass stars are still forming in the nascent globular cluster. They can, through the wind and SN shocks or through the ionization front they produce, trigger star formation in their vicinity, thus being able to be at the same time the cause of new star formation and the provider of at least part of the material from which the stars form. Moreover, it appears that rotating massive stars can lose low-speed material only enriched in H-burning products. Low- and intermediate-mass stars would not be able to do that, either because they are less efficiently mixed by rotation and/or they have more difficulty in reaching the break-up limit and of course the $\Omega \Gamma$-Limit than the massive stars during the main sequence. In addition their central temperature is too low to efficiently activate $\mathrm{NeNa}$ and $\mathrm{MgAl}$ chains during the MS. Also they require a more top-heavy IMF than massive stars, as explained by PC06.

Globular clusters may be suitable environments to form fast rotating massive stars in the proper range of metallicity. The WFRMS scenario to explain the GC abundance anomalies is only one aspect of study of fast rotating massive stars. This kind of star might be also useful to understand other features such

\footnotetext{
${ }^{10}$ Since massive stars are preferentially located in the central parts of a young cluster, they may have mainly polluted the central regions. The outer part of the GC progenitors would at least for a while be composed of non polluted stars which may have been stripped off by tidal effects.
}

as the origin of the carbon-rich ultra metal-poor stars, the high $\mathrm{N} / \mathrm{O}$ ratio observed in halo stars, and the high helium abundance of some of the stars in $\omega$ Cen (Maeder \& Meynet 2006). The anticorrelations observed in GC might thus be an additional observed consequences of the chemical enrichment expected from fast rotating massive stars.

Acknowledgements. The authors wish to thank Christian Iliadis for providing its reaction rates. T.D. and C.C. acknowledge financial support from French "Programme National de Physique stellaire".

\section{References}

Alexander, D. R., \& Ferguson, J. W. 1994, ApJ, 437, 879

Angulo, C., Arnould, M., Rayet, M., et al. 1999, Nuclear Physics A, 656, 3

Arnould, M., Goriely, S., \& Jorissen, A. 1999, A\&A, 347, 572

Brown, J. A., \& Wallerstein, G. 1993, AJ, 106, 133

Carretta, E., Bragaglia, A., Cacciari, C., \& Rossetti, E. 2003, A\&A, 410, 143

Carretta, E., Bragaglia, A., \& Cacciari, C. 2004, ApJ, 610, L25

Carretta, E., Gratton, R. G., Lucatello, S., Bragaglia, A., \& Bonifacio, P. 2005, A\&A, 433, 597

Chaboyer, B., \& Zahn, J.-P. 1992, A\&A, 253, 173

Charbonnel, C. 2005, in IAU Symp., 228, ed. V. Hill, P. François, \& F. Primas, 347

Coc, A., Vangioni-Flam, E., Descouvemont, P., Adahchour, A., \& Angulo, C. 2004, ApJ, 600, 544

Cohen, J. G., Briley, M. M., \& Stetson, P. B. 2002, AJ, 123, 2525

Cottrell, P. L., \& Da Costa, G. S. 1981, ApJ, 245, L79

de Jager, C., Nieuwenhuijzen, H., \& van der Hucht, K. A. 1988, A\&AS, 72, 259

Denissenkov, P. A., \& Herwig, F. 2003, ApJ, 590, L99

Dickens, R. J., Croke, B. F. W., Cannon, R. D., \& Bell, R. A. 1991, Nature, 351, 212

Dufton, P. L., Ryans, R. S. I., Simón-Díaz, S., Trundle, C., \& Lennon, D. J. 2006, A\&A, 451, 603

Fenner, Y., Campbell, S., Karakas, A. I., Lattanzio, J. C., \& Gibson, B. K. 2004, MNRAS, 353, 789

Freeman, K. C., \& Norris, J. 1981, ARA\&A, 19, 319

Freyer, T., Hensler, G., \& Yorke, H. W. 2003, ApJ, 594, 888

Garcia-Segura, G., Langer, N., \& Mac Low, M.-M. 1996, A\&A, 316, 133

Gnedin, O. Y., Zhao, H., Pringle, J. E., et al. 2002, ApJ, 568, L23

Gratton, R., Sneden, C., \& Carretta, E. 2004, ARA\&A, 42, 385

Gratton, R. G., Sneden, C., Carretta, E., \& Bragaglia, A. 2000, A\&A, 354, 169

Gratton, R. G., Bonifacio, P., Bragaglia, A., et al. 2001, A\&A, 369, 87

Grundahl, F., Briley, M., Nissen, P. E., \& Feltzing, S. 2002, A\&A, 385, L14

Hale, S. E., Champagne, A. E., Iliadis, C., et al. 2002, Phys. Rev. C, 65, 015801

Hale, S. E., Champagne, A. E., Iliadis, C., et al. 2004, Phys. Rev. C, 70, 045802

Harbeck, D., Smith, G. H., \& Grebel, E. K. 2003, AJ, 125, 197

Heger, A., \& Langer, N. 2000, ApJ, 544, 1016

Herwig, F. 2004a, ApJ, 605, 425

Herwig, F. 2004b, ApJS, 155, 651

Huang, W., \& Gies, D. R. 2006, ApJ, 648, 580

Iglesias, C. A., \& Rogers, F. J. 1996, ApJ, 464, 943

Iliadis, C., D’Auria, J. M., Starrfield, S., Thompson, W. J., \& Wiescher, M. 2001, ApJS, 134, 151

Ivans, I. I., Sneden, C., Kraft, R. P., et al. 1999, AJ, 118, 1273

James, G., François, P., Bonifacio, P., et al. 2004a, A\&A, 414, 1071

James, G., François, P., Bonifacio, P., et al. 2004b, A\&A, 427, 825

Karakas, A. I., \& Lattanzio, J. C. 2003, PASA, 20, 279

Kraft, R. P. 1994, PASP, 106, 553

Kraft, R. P., Sneden, C., Langer, G. E., \& Prosser, C. F. 1992, AJ, 104, 645

Kudritzki, R.-P., \& Puls, J. 2000, ARA\&A, 38, 613

Lamers, H. J. G. L. M., Snow, T. P., \& Lindholm, D. M. 1995, ApJ, 455, 269

Langer, N. 1998, A\&A, 329, 551

Maeda, K., \& Nomoto, K. 2003, ApJ, 598, 1163

Maeder, A. 1999, A\&A, 347, 185

Maeder, A., \& Meynet, G. 2000, A\&A, 361, 159

Maeder, A., \& Meynet, G. 2001, A\&A, 373, 555

Maeder, A., \& Meynet, G. 2006, A\&A, 448, L37

Maeder, A., \& Zahn, J.-P. 1998, A\&A, 334, 1000

Meynet, G., \& Maeder, A. 1997, A\&A, 321, 465

Meynet, G., \& Maeder, A. 2000, A\&A, 361, 101

Meynet, G., \& Maeder, A. 2002, A\&A, 390, 561 
Meynet, G., Ekström, S., Maeder, A., \& Barblan, F. 2006, ArXiv Astrophysics e-prints

Nugis, T., \& Lamers, H. J. G. L. M. 2000, A\&A, 360, 227

Pasquini, L., Bonifacio, P., Molaro, P., et al. 2005, A\&A, 441, 549

Porter, J. M., \& Rivinius, T. 2003, PASP, 115, 1153

Powell, D. C., Iliadis, C., Champagne, A. E., et al. 1999, Nucl. Phys. A, 660, 349

Prantzos, N., \& Charbonnel, C. 2006, A\&A, 458, 135

Ramírez, S. V., \& Cohen, J. G. 2002, AJ, 123, 3277

Ramírez, S. V., \& Cohen, J. G. 2003, AJ, 125, 224

Rivinius, T. 2005, in The Nature and Evolution of Disks Around Hot Stars, ed. R. Ignace, \& K. G. Gayley, ASP Conf. Ser., 337, 178

Sackmann, I.-J., \& Anand, S. P. S. 1970, ApJ, 162, 105

Salaris, M., Weiss, A., Ferguson, J. W., \& Fusilier, D. J. 2006, ArXiv Astrophysics e-prints

Shetrone, M. D. 1996, AJ, 112, 2639

Smith, G. H. 1987, PASP, 99, 67

Smith, G. H. 2006, PASP, in press

Sneden, C. 2005, in IAU Symposium, ed. V. Hill, P. François, \& F. Primas, 337
Sobeck, J. S., Ivans, I. I., Simmerer, J. A., et al. 2006, AJ, 131, 2949 Strom, S. E., Wolff, S. C., \& Dror, D. H. A. 2005, AJ, 129, 809

Talon, S. 2004, in IAU Symposium, ed. A. Maeder, \& P. Eenens, 336

Townsend, R. H. D., Owocki, S. P., \& Howarth, I. D. 2004, MNRAS, 350, 189

Ventura, P., \& D'Antona, F. 2005a, A\&A, 431, 279

Ventura, P., \& D’Antona, F. 2005b, A\&A, 439, 1075

Ventura, P., \& D'Antona, F. 2005c, ApJ, 635, L149

Ventura, P., \& D’Antona, F. 2006, A\&A, 457, 995

Ventura, P., D’Antona, F., Mazzitelli, I., \& Gratton, R. 2001, ApJ, 550, L65

Ventura, P., D’Antona, F., \& Mazzitelli, I. 2002, A\&A, 393, 215

Wallerstein, G., Myckky-Leep, E., \& Oke, J. B. 1987, AJ, 94, 523

Woltjer, L. 1975, A\&A, 42, 109

Yong, D., Grundahl, F., Lambert, D. L., Nissen, P. E., \& Shetrone, M. D. 2003, A\&A, 402, 985

Yong, D., Grundahl, F., Nissen, P. E., Jensen, H. R., \& Lambert, D. L. 2005, A\&A, 438, 875

Yong, D., Aoki, W., \& Lambert, D. L. 2006, ApJ, 638, 1018

Zahn, J.-P. 1992, A\&A, 265, 115 